\title{
ISOLATED ROUNDINGS AND FLATTENINGS OF SUBMANIFOLDS IN EUCLIDEAN SPACES
}

\author{
TOShizUmi FuKui AND JUAN J. NuÑo-BAllesteros
}

(Received May 30, 2003, revised May 11, 2005)

\begin{abstract}
We introduce the concepts of rounding and flattening of a smooth map $g$ of an $m$-dimensional manifold $M$ to the euclidean space $\boldsymbol{R}^{n}$ with $m<n$, as those points in $M$ such that the image $g(M)$ has contact of type $\Sigma^{m, \ldots, m}$ with a hypersphere or a hyperplane of $\boldsymbol{R}^{n}$, respectively. This includes several known special points such as vertices or flattenings of a curve in $\boldsymbol{R}^{n}$, umbilics of a surface in $\boldsymbol{R}^{3}$, or inflections of a surface in $\boldsymbol{R}^{4}$.
\end{abstract}

1. Introduction. In [24], Porteous studied the Thom-Boardman singularities $\Sigma^{i_{1}, \ldots, i_{k}}$ of the distance-squared unfolding associated with a smooth embedding $g: M \rightarrow \boldsymbol{R}^{n}$ from a smooth $m$-dimensional manifold $M$ into $\boldsymbol{R}^{n}$, which is defined by

$D: \boldsymbol{R}^{n} \times M \rightarrow \boldsymbol{R}^{n} \times \boldsymbol{R}, \quad(x, t) \mapsto\left(x, d_{x}(t)\right), \quad$ where $d_{x}(t)=(1 / 2)\|x-g(t)\|^{2}$.

He discovered that for small values of $m$ and $n$, these singularities give a new point of view of some well-known facts of classical differential geometry. This work was continued by Montaldi [19], who introduced the notion of contact between submanifolds and deduced that the singularity types of $D$ characterize the contact types of $g(M)$ with the hyperspheres of $\boldsymbol{R}^{n}$. It is known (see [32]) that for a residual set in $C^{\infty}\left(M, \boldsymbol{R}^{n}\right)$ with the Whitney $C^{\infty}$ topology, the associated unfolding $D$ is generic in the Thom-Boardman sense (that is, its $k$-jet extension is transverse to the strata $\left.\Sigma^{i_{1}, \ldots, i_{k}}\right)$.

For instance, suppose that $g: I \rightarrow \boldsymbol{R}^{2}$ defines a regular smooth curve in the plane. Then, a point of type $\Sigma^{1,1,1}$ of $D$ is a pair $(x, t)$ such that $t \in I$ is a vertex of the curve (that is, a point where $\kappa^{\prime}=0$ ) and $x \in \boldsymbol{R}^{2}$ is the center of curvature at that point. If $g: M^{2} \rightarrow \boldsymbol{R}^{3}$ is now a smooth immersion of a surface in $\boldsymbol{R}^{3}$, a point of type $\Sigma^{2,2}$ of $D$ is a pair $(x, t)$, where $t \in M$ is an umbilic (that is, the two principal curvatures coincide) and $x \in \boldsymbol{R}^{3}$ is the center of principal curvature.

We can do a similar analysis if we look at the height unfolding, given by

$$
H: S^{n-1} \times M \rightarrow S^{n-1} \times \boldsymbol{R}, \quad(v, t) \mapsto\left(v, h_{v}(t)\right), \quad \text { where } h_{v}(t)=\langle v, g(t)\rangle .
$$

In this case, the singularity types of $H$ determine the contact type of $g(M)$ with the hyperplanes of $\boldsymbol{R}^{n}$ and it is also true that for a residual set in $C^{\infty}\left(M, \boldsymbol{R}^{n}\right)$ the unfolding $H$ is generic in the Thom-Boardman sense. For instance, if $g: I \rightarrow \boldsymbol{R}^{3}$ is a regular curve with a well-defined Frenet frame, then the $\Sigma^{1,1,1}$ points of $H$ are the pairs $(v, t)$, where $t \in I$ is a flattening (that is, the torsion is zero at $t$ ) and $v \in S^{1}$ is the binormal vector. If $g: M^{2} \rightarrow \boldsymbol{R}^{4}$ is a smooth immersion of a surface in $\boldsymbol{R}^{4}$, a $\Sigma^{2,2}$ point of $H$ is a pair $(v, t)$, where $t \in M$ is

2000 Mathematics Subject Classification. Primary 53A07; Secondary 58K05, 53A05.

Key words and phrases. Distance squared function, height function. 
an inflection (that is, the two second fundamental forms are proportional) and $v \in S^{3}$ is the binormal vector in the sense of [8].

In recent years, these concepts have been of great interest for people working on differential geometry and singularities. On one hand, we have the classical four vertex theorem, which states that any closed simple regular plane curve has at least four vertices, and its generalization to higher dimensions (see, for instance, [1]). On the other hand, the Carathéodory conjecture states that every smooth immersion of the sphere $S^{2}$ into $\boldsymbol{R}^{3}$ has at least two umbilics (see Remark 4.7). Moreover, it has recently been shown (cf. [8]) that any convex generic immersion of the sphere $S^{2}$ into $\boldsymbol{R}^{4}$ has at least four inflections.

In Section 2, we introduce the concept of k-rounding (resp. k-flattening) as a point $t_{0} \in M$ which corresponds to a point of type $\Sigma^{m_{(k)}}$ of $D$ (resp. $H$ ). Here $m_{(k)}$ denotes the Boardman symbol $(m, \ldots, m)$, with $m$ repeated $k$ times. Note that these definitions make sense even when the map $g: M \rightarrow \boldsymbol{R}^{n}$ has singularities. Then, for each $m$ and $k$ we can choose $n$, the dimension of the ambient space, such that the $k$-roundings or the $k$-flattenings of a generic smooth map $g: M^{m} \rightarrow \boldsymbol{R}^{n}$ appear as isolated points (see Table 1).

Associated with a $k$-rounding or $k$-flattening we consider a local algebra of contact, which is obtained by eliminating the parameter $x$ or $v$ in the local algebra of contact of $D$ or $H$, respectively, with the stratum $\Sigma^{m_{(k)}}$. It follows that this local algebra of contact is a local geometric invariant (that is, it is invariant under change of coordinates in $M$ and isometries in $\boldsymbol{R}^{n}$ ). Moreover, from this algebra, we can also obtain numerical invariants such as its multiplicity or its index (in the orientable case). The index of a $k$-rounding or $k$-flattening is simply the oriented intersection number of the $k$-jet extension of the $d_{x}$ or $h_{v}$ with the stratum $\Sigma^{m_{(k)}}$. At the end of Section 2, we show that roundings and flattenings are related through the stereographic projection. We see that a point $t_{0} \in M$ is a rounding of $g: M \rightarrow \boldsymbol{R}^{n}$ if and only if it is a flattening of $\xi \circ g: M \rightarrow \boldsymbol{R}^{n+1}$, where $\xi: \boldsymbol{R}^{n} \rightarrow S^{n} \hookrightarrow \boldsymbol{R}^{n+1}$ denotes the inverse of the stereographic projection. Moreover, the index is also preserved.

In Section 3, we study curves in $\boldsymbol{R}^{n}$ and give an explicit formula to compute roundings (that is, vertices) which seems to be new.

Sections 4 and 5 are dedicated to surfaces in $\boldsymbol{R}^{3}$. In the case of an isolated umbilic of a surface in $\boldsymbol{R}^{3}$, we show that the index we have defined is essentially the index of the foliation defined by curvature lines. We also provide formulas for these indices which work even for isolated singular points of the surface. In Section 5, we introduce complex coordinates in regular surfaces, in order to compute indices of umbilic points by looking at the coefficients in a Monge normal form.

Finally, in Section 6 we study surfaces in $\boldsymbol{R}^{4}$. Then, we see that for an isolated inflection of a surface in $\boldsymbol{R}^{4}$, the index is essentially the index of the foliation defined by asymptotic lines. 


\section{Roundings and flattenings.}

2.1. Definitions of roundings and flattenings. Let $M$ be a smooth $m$-manifold and let $S_{k}$ denote the Thom-Boardman submanifold $\Sigma^{m_{(k)}}$ in the jet space $J^{r}(M, \boldsymbol{R}), k \leq r$, which is the subset of the jet space with all partial derivatives of degree $\leq k$ equal to zero.

Definition 2.1. Let $g: M \rightarrow \boldsymbol{R}^{n}$ be a smooth map from a smooth $m$-dimensional manifold $M$ into $\boldsymbol{R}^{n}$, with $m<n$. Let $k, r$ be the positive integers with $k \leq r$.

We say that $p \in M$ is a $k$-flattening of $g$ if there is $v \in S^{n-1}$ such that $j^{r} h_{v}(p) \in S_{k}$.

We say that $p \in M$ is a non-flat $k$-rounding of $g$ if it is not a $k$-flattening and there is $x \in \boldsymbol{R}^{n}$ such that $j^{r} d_{x}(p) \in S_{k}$.

We say that $p \in M$ is a $k$-rounding of $g$ if it is either a $k$-flattening or a non-flat $k$ rounding.

When $k$ is clear from the context, we will just say that $p$ is a flattening or a rounding.

Consider Thom-Boardman classes of the unfoldings $D(x, t)=\left(x, d_{x}(t)\right)$ and $H(v, t)=$ $\left(v, h_{v}(t)\right)$. Since they have corank one, we have that $(v, t) \in \Sigma^{m_{(k)}}(H)(\operatorname{resp} .(x, t) \in$ $\left.\Sigma^{m_{(k)}}(D)\right)$ if and only if $j^{r} h_{v}(p) \in S_{k}$ (resp. $\left.j^{r} d_{x}(p) \in S_{k}\right)$.

Assume that $g: M \rightarrow \boldsymbol{R}^{n}$ is an immersion. It follows from the definition that if $p \in M$ is a $k$-flattening of $g$ and $v \in S^{n-1}$ such that $(v, p) \in \Sigma^{m_{(k)}}(H)$, then $g(M)$ has a contact of type $\Sigma^{m_{(k)}}$ at $g(p)$ with the hyperplane of $\boldsymbol{R}^{n}$ orthogonal to $v$ through the point $g(p)$. Analogously, if $p$ is a non-flat $k$-rounding and $x \in \boldsymbol{R}^{n}$ such that $(x, p) \in \Sigma^{m_{(k)}}(D)$, then $g(M)$ has a contact of type $\Sigma^{m_{(k)}}$ at $g(p)$ with the hypersphere centered at $x$ and radius $R=\|x-g(p)\|$. Note that our definitions make sense even in the case that $g$ has singularities.

We now see that it is possible to eliminate the parameter $v$ or $x$ in the definition and obtain a condition just in terms of $p$. Let us denote it by $v=v\left(m_{(k)}\right)$ the cardinality of the set $\left\{\alpha=\left(\alpha_{1}, \ldots, \alpha_{m}\right) ; 1 \leq|\alpha| \leq k\right\}$, so that $v$ gives exactly the number of local equations which define $\Sigma^{m_{(k)}}$. This number can easily be computed by the combinatorial formula

$$
v=\left(\begin{array}{c}
m+k \\
m
\end{array}\right)-1
$$

Moreover, we will fix some order in this set (for instance, the degree lexicographical order associated with the monomials $t^{\alpha}$ ).

Definition 2.2. Let $g: M \rightarrow \boldsymbol{R}^{n}$ be a smooth map. Given a local coordinate system $\phi: U \subset M \rightarrow \boldsymbol{R}^{m}$, we denote $\tilde{g}=g \circ \phi^{-1}$ and $\phi(p)=t$, for each $p \in U$. We define the matrices

$$
F^{k}(\tilde{g} ; p)=\left(\frac{\partial^{|\alpha|} \tilde{g}_{i}}{\partial t^{\alpha}}(t)\right)_{\substack{1 \leq i \leq n \\ 1 \leq|\alpha| \leq k}} \text { and } R^{k}(\tilde{g} ; p)=\left(\frac{\partial^{|\alpha|} \tilde{g}_{i}}{\partial t^{\alpha}}(t) \mid \varphi_{\alpha}(t)\right)_{\substack{1 \leq i \leq n \\ 1 \leq|\alpha| \leq k}},
$$

where the functions $\varphi_{\alpha}$ are defined by induction on $|\alpha|$ in the following way. If $|\alpha|=1$, then $\varphi_{\alpha}=0$. If $|\alpha|>1$, there are $\beta$ and $i \in\{1, \ldots, m\}$ such that $\alpha=\beta+\left(0, \ldots, 1^{i)}, \ldots, 0\right)$; then,

$$
\varphi_{\alpha}=\left\langle\frac{\partial \tilde{g}}{\partial t_{i}}, \frac{\partial^{|\beta|} \tilde{g}}{\partial t^{\beta}}\right\rangle+\frac{\partial \varphi_{\beta}}{\partial t_{i}}
$$


The matrices $F^{k}(\tilde{g} ; p)$ and $R^{k}(\tilde{g} ; p)$ have size $v \times n$ and $v \times(n+1)$, respectively. Again, when $k$ is clear from the context, we shall denote the matrices just by $F(\tilde{g} ; p)$ and $R(\tilde{g} ; p)$.

LeMMA 2.3. Let $g: M \rightarrow \boldsymbol{R}^{n}$ be a smooth map and let $p \in M$. Then:

(1) $p$ is a k-flattening of $g$ if and only if $\operatorname{rank} F^{k}(\tilde{g} ; p)<n$;

(2) $p$ is a k-rounding of $g$ if and only if $\operatorname{rank} R^{k}(\tilde{g} ; p)<n+1$.

Proof. Since $h_{v} \circ \phi^{-1}(t)=\langle v, \tilde{g}(t)\rangle$, we have

$$
\frac{\partial^{|\alpha|}\left(h_{v} \circ \phi^{-1}\right)}{\partial t^{\alpha}}(t)=\left\langle v, \frac{\partial^{|\alpha|} \tilde{g}}{\partial t^{\alpha}}(t)\right\rangle,
$$

so that $p$ is a $k$-flattening if and only if there is $v \in S^{n-1}$ such that

$$
\left\langle v, \frac{\partial^{|\alpha|} \tilde{g}}{\partial t^{\alpha}}(t)\right\rangle=0, \quad \forall \alpha: 1 \leq|\alpha| \leq k .
$$

This is a homogeneous linear system in the variables $v_{1}, \ldots, v_{n}$, whose coefficient matrix is $F(\tilde{g} ; p)$. It follows that there is a solution $v \in S^{n-1}$ if and only if $F^{k}(\tilde{g} ; p)$ has rank $<n$.

For the distance-squared functions, we have that $d_{x} \circ \phi^{-1}(t)=(1 / 2)\|x-\tilde{g}(t)\|^{2}$ and, hence,

$$
\frac{\partial^{|\alpha|}\left(d_{x} \circ \phi^{-1}\right)}{\partial t^{\alpha}}(t)=-\left\langle x-\tilde{g}(t), \frac{\partial^{|\alpha|} \tilde{g}}{\partial t^{\alpha}}(t)\right\rangle+\varphi_{\alpha}(t) .
$$

Now, $p$ is a non-flat $k$-rounding if and only if it is not a $k$-flattening and there is $x \in \boldsymbol{R}^{n}$ such that

$$
\left\langle x-\tilde{g}(t), \frac{\partial^{|\alpha|} \tilde{g}}{\partial t^{\alpha}}(t)\right\rangle=\varphi_{\alpha}(t), \quad \forall \alpha: 1 \leq|\alpha| \leq k .
$$

In this case, we have a non-homogeneous linear system in the variables $X_{i}=x_{i}-g_{i}(t)$, $i=1, \ldots, n$, with coefficient matrix $F^{k}(\tilde{g} ; p)$ and with independent terms $\left(\varphi_{\alpha}(t)\right)$. It follows that the system has a solution if and only if $\operatorname{rank} F^{k}(\tilde{g} ; p)=\operatorname{rank} R^{k}(\tilde{g} ; p)=n$.

Finally, note that

$$
\operatorname{rank} F^{k}(\tilde{g} ; p) \leq \operatorname{rank} R^{k}(\tilde{g} ; p) \leq \operatorname{rank} F^{k}(\tilde{g} ; p)+1,
$$

which completes the proof.

It follows from the proof of the above lemma that if $p \in M$ is a non-flat $k$-rounding of $g: M \rightarrow \boldsymbol{R}^{n}$, then there is a unique $x \in \boldsymbol{R}^{n}$ such that $j^{k} d_{x}(p) \in S_{k}$ (i.e., $\left.(x, p) \in \Sigma^{m_{(k)}}(D)\right)$.

EXAMPLE 2.4. In the following sections, we will see that roundings and flattenings coincide with classical concepts in differential geometry of curves and surfaces.

(1) Let $\alpha: I \rightarrow \boldsymbol{R}^{2}$ be a regular plane curve. Then $t \in I$ is a 2 -flattening if and only if it is an inflection (that is, the curvature $\kappa$ is zero at $t$ ). The vertices of $\alpha$ (that is, the points where $\kappa^{\prime}(t)=0$ ) correspond to the 3-roundings of $\alpha$.

(2) Suppose now that $\alpha: I \rightarrow \boldsymbol{R}^{3}$ is a regular space curve with non-vanishing curvature, so that it has a well-defined Frenet frame. Then, a 3 -flattening is a point where the torsion $\tau$ is zero. 
(3) Let $g: M^{2} \rightarrow \boldsymbol{R}^{3}$ be a regular surface in $\boldsymbol{R}^{3}$. Then 2-roundings correspond to umbilic points of $g$ (that is, points where the two principal curvatures are equal).

(4) Finally, if $g: M^{2} \rightarrow \boldsymbol{R}^{4}$ is a regular surface in $\boldsymbol{R}^{4}$, the 2-flattenings of $g$ are known as inflections and correspond to points where the two second fundamental forms are linearly dependent.

Definition 2.5. Let $g: U \subset \boldsymbol{R}^{m} \rightarrow \boldsymbol{R}^{n}$ be a smooth map. Given $t_{0} \in U$ we denote by $C^{\infty}\left(\boldsymbol{R}^{m}, t_{0}\right)$ the local algebra of smooth function germs from $\left(\boldsymbol{R}^{m}, t_{0}\right)$ to $\boldsymbol{R}$. Then, we define $\mathcal{R}\left(g, t_{0}\right)$ as the ideal in $C^{\infty}\left(\boldsymbol{R}^{m}, t_{0}\right)$ generated by the germs at $t_{0}$ of the $(n+1)$-minors of $R(g, t)$.

Analogously, we can define $\mathcal{F}\left(g, t_{0}\right)$ as the ideal in $C^{\infty}\left(\boldsymbol{R}^{m}, t_{0}\right)$ generated by the germs at $t_{0}$ of the $n$-minors of $F(g, t)$.

The following lemma shows that although the matrices $R(g, t)$ and $F(g, t)$ depend on the choice of coordinates in $\boldsymbol{R}^{m}$, the corresponding ideals $\mathcal{R}\left(g, t_{0}\right)$ and $\mathcal{F}\left(g, t_{0}\right)$ are coordinate independent.

LEMMA 2.6. Let $g: U \subset \boldsymbol{R}^{m} \rightarrow \boldsymbol{R}^{n}$ be a smooth map and $t_{0} \in U$. Suppose that $\phi:\left(\boldsymbol{R}^{m}, t_{0}^{\prime}\right) \rightarrow\left(\boldsymbol{R}^{m}, t_{0}\right)$ is a diffeomorphism germ and denote by $\phi^{*}: C^{\infty}\left(\boldsymbol{R}^{m}, t_{0}\right) \rightarrow$ $C^{\infty}\left(\boldsymbol{R}^{m}, t_{0}^{\prime}\right)$ the induced isomorphism of local algebras. Then, $\phi^{*}\left(\mathcal{R}\left(g, t_{0}\right)\right)=\mathcal{R}\left(g \circ \phi, t_{0}^{\prime}\right)$ and $\phi^{*}\left(\mathcal{F}\left(g, t_{0}\right)\right)=\mathcal{F}\left(g \circ \phi, t_{0}^{\prime}\right)$.

Proof. Let us denote by $e_{i}$ the $m$-tuple $\left(0, \ldots, 1^{i}, \ldots, 0\right)$. We define the function germs $\lambda_{\alpha \beta} \in C^{\infty}\left(\boldsymbol{R}^{m}, t_{0}^{\prime}\right)$, with $1 \leq|\beta| \leq|\alpha|$ by induction on $|\alpha|$ in the following way:

$$
\lambda_{e_{i}, e_{j}}=\frac{\partial \phi_{j}}{\partial t_{i}}
$$

and if $|\alpha|>1$ and $|\alpha| \geq|\beta|$, then

$$
\lambda_{\alpha+e_{i}, \beta}=\frac{\partial \lambda_{\alpha \beta}}{\partial t_{i}}+\sum_{j=1}^{m} \lambda_{e_{i}, e_{j}} \lambda_{\alpha, \beta-e_{j}}
$$

where we take the convention that $\lambda_{\alpha \beta}=0$ when it is not defined. Then, by direct application of the chain rule we obtain that for any $\alpha$, with $|\alpha|=r$,

$$
\frac{\partial^{r}(g \circ \phi)}{\partial t^{\alpha}}=\sum_{1 \leq|\beta| \leq r} \lambda_{\alpha \beta}\left(\frac{\partial^{|\beta|} g}{\partial t^{\beta}} \circ \phi\right) .
$$

In fact, we have

$$
\begin{aligned}
\frac{\partial^{r+1}(g \circ \phi)}{\partial t^{\alpha+e_{i}}} & =\frac{\partial}{\partial t_{i}}\left(\sum_{\beta} \lambda_{\alpha \beta}\left(\frac{\partial^{|\beta|} g}{\partial t^{\beta}} \circ \phi\right)\right) \\
& =\sum_{\beta} \frac{\partial \lambda_{\alpha \beta}}{\partial t_{i}} \frac{\partial^{|\beta|} g}{\partial t^{\beta}} \circ \phi+\sum_{\beta}\left(\lambda_{\alpha \beta} \sum_{j}\left(\frac{\partial^{|\beta|+1} g}{\partial t^{\beta+e_{j}}} \circ \phi\right) \frac{\partial \phi_{j}}{\partial t_{j}}\right)
\end{aligned}
$$




$$
\begin{aligned}
& =\sum_{\beta} \frac{\partial \lambda_{\alpha \beta}}{\partial t_{i}} \frac{\partial^{|\beta|} g}{\partial t^{\beta}} \circ \phi+\sum_{\beta}\left(\sum_{j} \lambda_{\alpha \beta-e_{j}} \lambda_{e_{i}, e_{j}}\left(\frac{\partial^{|\beta|} g}{\partial t^{\beta}} \circ \phi\right)\right) \\
& =\sum_{\beta} \lambda_{\alpha+e_{i}, \beta}\left(\frac{\partial^{|\beta|} g}{\partial t^{\beta}} \circ \phi\right) .
\end{aligned}
$$

This implies that any $n$-minor $d$ of $F^{k}(g \circ \phi, t)$ can be written as a linear combination $d=$ $\sum a_{i} \phi^{*}\left(d_{i}\right)$, where $d_{i}$ are $n$-minors of $F^{k}(g, t)$ and $a_{i} \in C^{\infty}\left(\boldsymbol{R}^{m}, t_{0}^{\prime}\right)$. This gives the inclusion $\mathcal{F}\left(g \circ \phi, t_{0}^{\prime}\right) \subset \phi^{*}\left(\mathcal{F}\left(g, t_{0}\right)\right)$ and the opposite inclusion follows by applying the same argument to $\tilde{g}=g \circ \phi$ and $\tilde{\phi}=\phi^{-1}$.

To conclude that $\phi^{*}\left(\mathcal{R}\left(g, t_{0}\right)\right)=\mathcal{R}\left(g \circ \phi, t_{0}^{\prime}\right)$, we just need to prove that

$$
\varphi_{\alpha}^{\prime}=\sum_{1 \leq|\beta| \leq r} \lambda_{\alpha \beta}\left(\varphi_{\beta} \circ \phi\right),
$$

where $\varphi_{\alpha}^{\prime}$ are the corresponding functions for $g \circ \phi$. Then, (2.3) follows from (2.2) and (2.1) by induction on $r=|\alpha|$ :

$$
\begin{aligned}
\varphi_{\alpha+e_{i}}^{\prime} & =\left\langle\frac{\partial(g \circ \phi)}{\partial t_{i}}, \frac{\partial^{r}(g \circ \phi)}{\partial t^{\alpha}}\right\rangle+\frac{\partial \varphi_{\alpha}^{\prime}}{\partial t_{i}} \\
& =\sum_{j, \beta} \lambda_{e_{i}, e_{j}} \lambda_{\alpha \beta}\left\langle\frac{\partial g}{\partial t_{j}} \circ \phi, \frac{\partial^{|\beta|} g}{\partial t^{\beta}} \circ \phi\right\rangle+\sum_{\beta} \frac{\partial \lambda_{\alpha \beta}}{\partial t_{i}}\left(\varphi_{\beta} \circ \phi\right)+\sum_{j, \beta} \lambda_{e_{i}, e_{j}} \lambda_{\alpha \beta}\left(\frac{\partial \varphi_{\beta}}{\partial t_{j}} \circ \phi\right) \\
& =\sum_{j, \beta} \lambda_{e_{i}, e_{j}} \lambda_{\alpha \beta}\left(\left\langle\frac{\partial g}{\partial t_{j}} \circ \phi, \frac{\partial^{|\beta|} g}{\partial t^{\beta}} \circ \phi\right\rangle+\frac{\partial \varphi_{\beta}}{\partial t_{j}} \circ \phi\right)+\sum_{\beta} \frac{\partial \lambda_{\alpha \beta}}{\partial t_{i}}\left(\varphi_{\beta} \circ \phi\right) \\
& =\sum_{j, \beta} \lambda_{e_{i}, e_{j}} \lambda_{\alpha \beta}\left(\varphi_{\beta+e_{j}} \circ \phi\right)+\sum_{\beta} \frac{\partial \lambda_{\alpha \beta}}{\partial t_{i}}\left(\varphi_{\beta} \circ \phi\right) \\
& =\sum_{j, \beta} \lambda_{e_{i}, e_{j}} \lambda_{\alpha, \beta-e_{j}}\left(\varphi_{\beta} \circ \phi\right)+\sum_{\beta} \frac{\partial \lambda_{\alpha \beta}}{\partial t_{i}}\left(\varphi_{\beta} \circ \phi\right) \\
& =\sum_{\beta} \lambda_{\alpha+e_{i}, \beta}\left(\varphi_{\beta} \circ \phi\right) .
\end{aligned}
$$

2.2. Multiplicities of flattenings and roundings. Let $g: M \rightarrow \boldsymbol{R}^{n}$ be a smooth map from a smooth $m$-dimensional manifold $M$ into $\boldsymbol{R}^{n}$. Given $p \in M$, we denote by $C^{\infty}(M, p)$ the local algebra of smooth function germs from $(M, p)$ into $\boldsymbol{R}$. If we choose a local chart $\phi:(M, p) \rightarrow\left(\boldsymbol{R}^{m}, t_{0}\right)$, we can consider the ideals $\mathcal{R}\left(g \circ \phi^{-1}, t_{0}\right)$ and $\mathcal{F}\left(g \circ \phi^{-1}, t_{0}\right)$ in $C^{\infty}\left(\boldsymbol{R}^{m}, t_{0}\right)$. We define the ideals $\mathcal{R}(g, p)$ and $\mathcal{F}(g, p)$ in $C^{\infty}(M, p)$ by

$$
\mathcal{R}(g, p)=\phi^{*} \mathcal{R}\left(g \circ \phi^{-1}, t_{0}\right), \quad \mathcal{F}(g, p)=\phi^{*} \mathcal{F}\left(g \circ \phi^{-1}, t_{0}\right),
$$

where $\phi^{*}: C^{\infty}\left(\boldsymbol{R}^{m}, t_{0}\right) \rightarrow C^{\infty}(M, p)$ is the induced isomorphism of local algebras. The above lemma easily shows that this construction does not depend on the chosen chart $\phi$. 
We now define the multiplicity of a rounding or a flattening, respectively, as

$\mu_{\mathcal{R}}(g, p)=\operatorname{dim}_{\boldsymbol{R}} C^{\infty}(M, p) / \mathcal{R}(g, p), \quad \mu_{\mathcal{F}}(g, p)=\operatorname{dim}_{\boldsymbol{R}} C^{\infty}(M, p) / \mathcal{F}(g, p)$.

If $p \in M$ is in fact a rounding or a flattening, we have that $\mu_{\mathcal{R}}(g, p) \geq 1$ or $\mu_{\mathcal{F}}(g, p) \geq 1$, respectively. Moreover, it is also true that if $\mu_{\mathcal{R}}(g, p)<\infty$ or $\mu_{\mathcal{F}}(g, p)<\infty$, then $p$ is an isolated rounding or flattening, respectively.

Now we recall the concept of multiplicity of a map with respect to a submanifold. Let $f: M \rightarrow N$ be a smooth map between manifolds and $S \subset N$ a submanifold such that $\operatorname{dim} M=\operatorname{codim} S$. Given $p \in M$ such that $f(p) \in S$, the multiplicity of $f$ and $S$ at $p$ is equal to

$$
m_{p}(f, S)=\operatorname{dim}_{R} C^{\infty}(M, p) / f^{*} I_{S},
$$

where $I_{S}$ is the ideal in $C^{\infty}(N, f(p))$ given by the function germs vanishing on $S$ and $f^{*}$ : $C^{\infty}(N, f(p)) \rightarrow C^{\infty}(M, p)$ is the induced homomorphism of local algebras.

Below we give some basic properties of the multiplicities.

(1) $m_{p}(f, S) \geq 1$ and the equality holds if and only if $f$ is transverse to $S$ at $p$.

(2) If $m_{p}(f, S)<\infty$, then $p$ is an isolated point of $f^{-1}(S)$.

(3) Assume that $S$ is locally given near $f(p)$ by $S=g^{-1}(0)$, where $g:(N, f(p)) \rightarrow$ $\left(\boldsymbol{R}^{m}, 0\right)$ is a smooth map germ with 0 as a regular value. Then $f^{*} I_{S}$ is generated by the components of the map germ $g \circ f:(M, p) \rightarrow\left(\boldsymbol{R}^{m}, 0\right)$, and, hence, $m_{p}(f, S)$ is equal to the multiplicity of $g \circ f$.

LEMMA 2.7. Let $g: M \rightarrow \boldsymbol{R}^{n}$ be a smooth map from a smooth $m$-dimensional manifold $M$ into $\boldsymbol{R}^{n}$. Suppose that $v=v\left(m_{(k)}\right)=n+m$ and that $p \in M$ is a non-flat rounding of $g$ and let $x_{0} \in \boldsymbol{R}^{n}$ be such that $\left(x_{0}, p\right) \in \Sigma^{m_{(k)}}(D)$. Then if $v=v\left(m_{(k)}\right)=n+m$, then

$$
\mu_{\mathcal{R}}(g, p)=m_{\left(x_{0}, p\right)}\left(j^{k} D, \Sigma^{m_{(k)}}\right) .
$$

Analogously, suppose that $v=v\left(m_{(k)}\right)=n+m-1$ and that $p \in M$ is a flattening of rank $n-1$ of $g$ and let $v_{0} \in S^{n-1}$ such that $\left(v_{0}, p\right) \in \Sigma^{m_{(k)}}(H)$. Then

$$
\mu_{\mathcal{F}}(g, p)=m_{\left(v_{0}, p\right)}\left(j^{k} H, \Sigma^{m_{(k)}}\right) .
$$

ProOF. By taking coordinates in $M$ we can suppose that $M=U \subset \boldsymbol{R}^{m}$, an open subset. Then, $\mathcal{R}(g, p)$ is the ideal in $C^{\infty}\left(\boldsymbol{R}^{m}, p\right)$ generated by the maximal minors of $R(g, p)$.

Since the rounding $p$ is non-flat, the matrix $R(g, p)$ has rank $n$ and we can suppose, without loss of generality, that the first $n$ rows of $R(g, p)$ are linearly independent. In this way, we can write

$$
R\left(g, t_{0}\right)=\left(\begin{array}{ll}
P & R \\
Q & S
\end{array}\right)
$$

where $P$ is an invertible matrix of size $n \times n, Q$ has size $(\nu-n) \times n$ and $R, S$ have size $n \times 1$ and $(v-n) \times 1$, respectively. Let $\Lambda$ be the $v \times v$ invertible matrix given by

$$
\Lambda=\left(\begin{array}{cc}
P^{-1} & 0 \\
-Q P^{-1} & I
\end{array}\right),
$$


where $I$ denotes the identity matrix of the corresponding size. Then, $\mathcal{R}(g, p)$ is also generated by the maximal minors of the matrix

$$
\Lambda R\left(g, t_{0}\right)=\left(\begin{array}{cc}
P^{-1} & 0 \\
-Q P^{-1} & I
\end{array}\right)\left(\begin{array}{ll}
P & R \\
Q & S
\end{array}\right)=\left(\begin{array}{cc}
I & 0 \\
0 & -Q P^{-1} R+S
\end{array}\right) .
$$

In particular, this gives that $R(g, p)$ is the ideal generated by the $m$ components of the matrix $-Q P^{-1} R+S$.

On the other hand, according to the third property of the multiplicity, $J=\left(j^{k} D\right)^{*} I_{\Sigma^{m}(k)}$ is the ideal in $C^{\infty}\left(\boldsymbol{R}^{n} \times \boldsymbol{R}^{m},\left(x_{0}, p\right)\right)$ generated by the $v$ components of the matrix

$$
\left(\begin{array}{l}
P \\
Q
\end{array}\right) X+\left(\begin{array}{l}
R \\
S
\end{array}\right)
$$

where $X$ is the column matrix with components $X_{i}=x_{i}-g_{i}(t), i=1, \ldots, n$. Again, we can take the product with the invertible matrix $\Lambda$. That is, $J$ is also generated by the $v$ components of the matrix

$$
\left(\begin{array}{cc}
P^{-1} & 0 \\
-Q P^{-1} & I
\end{array}\right)\left(\left(\begin{array}{l}
P \\
Q
\end{array}\right) X+\left(\begin{array}{l}
R \\
S
\end{array}\right)\right)=\left(\begin{array}{c}
X+P^{-1} R \\
-Q P^{-1} R+S
\end{array}\right) .
$$

Therefore, we can easily conclude that the local algebra $C^{\infty}\left(\boldsymbol{R}^{n} \times \boldsymbol{R}^{m},\left(x_{0}, t_{0}\right)\right) / J$ is isomorphic to $C^{\infty}\left(\boldsymbol{R}^{m}, t_{0}\right) / \mathcal{R}\left(g, t_{0}\right)$.

The proof of the second part of the lemma is analogous.

Definition 2.8. Let $g: M \rightarrow \boldsymbol{R}^{n}$ be a smooth map from a smooth $m$-dimensional manifold $M$ into $\boldsymbol{R}^{n}$. We say that $g$ is generic if the unfoldings $D, H$ are generic in the Thom-Boardman sense (that is, their $k$-jet extensions are transverse to the Thom-Boardman strata). In particular, it follows that $\Sigma^{m_{(k)}}(D)$ and $\Sigma^{m_{(k)}}(H)$ are submanifolds of codimension $v=v\left(m_{(k)}\right)$ of $\boldsymbol{R}^{n} \times M$ and $S^{n-1} \times M$, respectively. Thus:

(1) if $v=n+m$ and $g$ is generic, the roundings of $g$ are non-flat, appear as isolated points and have multiplicity one;

(2) if $v=n+m-1$ and $g$ is generic, the flattenings of $g$ have rank $n-1$, appear as isolated points and have multiplicity one.

See [32] for the proof of the generality of the generic map; that is, any map can be approximated by a sequence of generic maps.

In Table 1, we show the value of $n=v\left(m_{(k)}\right)-m$, so that roundings of a map $g$ : $M^{m} \rightarrow \boldsymbol{R}^{n}$ appear generically as isolated points. By adding one to all the entries of the table, we get $n=v\left(m_{(k)}\right)-m+1$, which corresponds to the dimensions where flattenings appear generically as isolated points.

When the map $g: M \rightarrow \boldsymbol{R}^{n}$ is not generic, we can take a generic deformation $g_{\lambda}:$ $M \rightarrow \boldsymbol{R}^{n}, \lambda \in(-\varepsilon, \varepsilon)$, which means that $g_{0}=g, g_{\lambda}$ is generic for $\lambda \neq 0$ and the map $(\lambda, t) \mapsto g_{\lambda}(t)$ is smooth. If $t_{0} \in M$ is a rounding or a flattening of $g$, we can ask about the number of roundings or flattenings that appear in $g_{\lambda}$ near $p$, for $\lambda \neq 0$ small enough. We will denote these numbers by $n_{\mathcal{R}}\left(g_{\lambda}, p\right)$ or $n_{\mathcal{F}}\left(g_{\lambda}, p\right)$, respectively. In the following theorem, we see that in the analytic case, such numbers are related to the corresponding multiplicities. 
TABLE 1.

\begin{tabular}{rrrrrrrrrr}
\hline & \multicolumn{10}{c}{$k$} \\
\cline { 2 - 10 }$m$ & 2 & 3 & 4 & 5 & 6 & 7 & 8 & 9 & 10 \\
\hline 1 & 1 & 2 & 3 & 4 & 5 & 6 & 7 & 8 & 9 \\
2 & 3 & 7 & 12 & 18 & 25 & 33 & 42 & 52 & 63 \\
3 & 6 & 16 & 31 & 52 & 80 & 116 & 161 & 216 & 282 \\
4 & 10 & 30 & 65 & 121 & 205 & 325 & 490 & 710 & 996 \\
5 & 15 & 50 & 120 & 246 & 456 & 786 & 1281 & 1996 & 2997 \\
\hline
\end{tabular}

THEOREM 2.9. Suppose that $g: M^{m} \rightarrow \boldsymbol{R}^{n}$ is analytic and let $g_{\lambda}: M \rightarrow \boldsymbol{R}^{n}$, $\lambda \in(-\varepsilon, \varepsilon)$, be an analytic generic deformation of $g$.

(1) If $p \in M$ is a rounding of finite multiplicity and $v\left(m_{(k)}\right)=n+m$, then $n_{\mathcal{R}}\left(g_{\lambda}, p\right) \leq$ $\mu_{\mathcal{R}}(g, p)$ and $n_{\mathcal{R}}\left(g_{\lambda}, p\right) \equiv \mu_{\mathcal{R}}(g, p)$ modulo 2 , for $\lambda \neq 0$ small enough.

(2) If $p \in M$ is a flattening of finite multiplicity and $v\left(m_{(k)}\right)=n+m-1$, then $n_{\mathcal{F}}\left(g_{\lambda}, p\right) \leq \mu_{\mathcal{F}}(g, p)$ and $n_{\mathcal{F}}\left(g_{\lambda}, p\right) \equiv \mu_{\mathcal{F}}(g, p)$ modulo 2 , for $\lambda \neq 0$ small enough.

Proof. As usual, by taking coordinates we can suppose that $M=U$ is an open subset of $\boldsymbol{R}^{m}$. Then, $g: U \rightarrow \boldsymbol{R}^{n}$ induces a complex analytic map $\hat{g}: \hat{U} \rightarrow \boldsymbol{C}^{n}$, where $\hat{U}$ is an open neighborhood of $p$ in $\boldsymbol{C}^{m}$. We will denote by $\mathcal{O}_{\boldsymbol{C}^{m}, p}$ the local ring of complex analytic function germs from $\left(\boldsymbol{C}^{m}, p\right)$ to $\boldsymbol{C}$, and $\mathcal{R}(\hat{g}, p)$ will denote the ideal in $\mathcal{O}_{\boldsymbol{C}^{m}}, p$ generated by the maximal minors of the matrix $R(\hat{g}, t)$ defined in an obvious way. Moreover, it follows that

so that

$$
\mathcal{O}_{C^{m}, p} / \mathcal{R}(\hat{g}, p) \cong C^{\infty}\left(\boldsymbol{R}^{m}, p\right) / \mathcal{R}(g, p) \otimes C
$$

$$
\mu_{\mathcal{R}}(g, p)=\operatorname{dim}_{\boldsymbol{R}} C^{\infty}\left(\boldsymbol{R}^{m}, p\right) / \mathcal{R}(g, p)=\operatorname{dim}_{\boldsymbol{C}} \mathcal{O}_{\boldsymbol{C}^{m}, p} / \mathcal{R}(\hat{g}, p) .
$$

On the other hand, the deformation $g_{\lambda}: U \rightarrow \boldsymbol{R}^{n}$ also induces a complex analytic deformation $\hat{g}_{\lambda}: \hat{U} \rightarrow \boldsymbol{C}^{n}, \lambda \in \hat{V}$, where now $\hat{V}$ is an open neighborhood of 0 in $\boldsymbol{C}$. We will denote by $n_{\mathcal{R}}\left(\hat{g}_{\lambda}, p\right)$ the number of complex roundings of $\hat{g}_{\lambda}$ near $p$. It follows that $n_{\mathcal{R}}\left(g_{\lambda}, p\right) \leq n_{\mathcal{R}}\left(\hat{g}_{\lambda}, p\right)$ and $n_{\mathcal{R}}\left(g_{\lambda}, p\right) \equiv n_{\mathcal{R}}\left(\hat{g}_{\lambda}, p\right)$ and, thus, it is enough to prove that for $\lambda \neq 0$ small enough,

$$
n_{\mathcal{R}}\left(\hat{g}_{\lambda}, p\right)=\operatorname{dim}_{\boldsymbol{C}} \mathcal{O}_{C^{m}, p} / \mathcal{R}(\hat{g}, p) .
$$

To see this, we use classical arguments of complex analytic geometry. To simplify notation, let us denote by $A$ the ring $\mathcal{O}_{\boldsymbol{C} \times \boldsymbol{C}^{m}},(0, p)$ and by $I$ the ideal in $A$ generated by the maximal minors of the matrix $R\left(\hat{g}_{\lambda}, t\right)$. The complex analytic set germ of zeros of $I, V(I)$, is given by the pairs $(\lambda, t)$ in a neighborhood of $(0, p)$ such that $t$ is a rounding of $g_{\lambda}$. Thus, the number $n_{\mathcal{R}}\left(\hat{g}_{\lambda}, p\right)$ is equal to the cardinality of $\pi^{-1}(\lambda)$, where $\pi$ is the finite map germ $\pi: V(I) \rightarrow C$, given by $\pi(\lambda, t)=\lambda$. By the Samuel formula (see, for instance, [22]), $\pi^{-1}(\lambda)$ for $\lambda \neq 0$ small enough, is equal to the multiplicity $e(\langle\bar{\lambda}\rangle ; A / I)$, where $\bar{\lambda}$ is the class of $\lambda$ in $A / I$. 
Now, $I$ is defined by the maximal minors of a matrix of size $v \times(n+1)$ and a result by Hochster and Eagon [12] ensures that $V(I)$ has dimension $\geq m+1-(v-(n+1)+1)=1$ and if the equality holds, then $A / I$ is a Cohen-Macaulay ring. Note that the finiteness of $\pi$ implies that the dimension of $V(I)$ is $\leq 1$. Thus, $V(I)$ is, in fact, one-dimensional and $A / I$ is Cohen-Macaulay.

Finally, use Theorem 17.11 of [17] to conclude that since $A / I$ is a one-dimensional Cohen-Macaulay ring and $\bar{\lambda}$ is a parameter system in $A / I$, then

$$
e(\langle\bar{\lambda}\rangle ; A / I)=\operatorname{dim}_{C}(A / I) /\langle\bar{\lambda}\rangle=\operatorname{dim}_{\boldsymbol{C}} \mathcal{O}_{C^{m}, p} / \mathcal{R}(\hat{g}, p) .
$$

The proof of the second part of the theorem is exactly the same.

2.3. Oriented intersection numbers and indices. Let $M$ be an oriented manifold and let $S$ be a co-oriented submanifold of a manifold $N$ with $\operatorname{dim} M=\operatorname{codim} S$. Assume that $f:(M, p) \rightarrow(N, f(p))$ is a smooth map germ with $f^{-1}(S)=p$. We denote the oriented intersection number of $f$ and $S$ at $p$ by $I_{p}(f, S)$.

Some basic properties of the oriented intersection number are the following.

(1) If $f$ is transverse to $S$ at $p$, then $I_{p}(f, S)= \pm 1$.

(2) Assume that $S$ is locally given near $f(p)$ by $S=g^{-1}(0)$, where $g:(N, f(p)) \rightarrow$ $\left(\boldsymbol{R}^{m}, 0\right)$ is a smooth map germ with 0 as a regular value so that the co-orientation of $S$ in $N$ coincides with the orientation of $\boldsymbol{R}^{m}$ via $g$. Then $I_{p}(f, S)$ is equal to the mapping degree of the map germ $g \circ f:(M, p) \rightarrow\left(\boldsymbol{R}^{m}, 0\right)$.

We now introduce the concept of index of a non-flat isolated rounding or an isolated flattening of rank $n-1$, which can be seen as an oriented version of multiplicity. Note that if $M$ is an oriented manifold, we have an induced orientation in $J^{k}(M, \boldsymbol{R})$. Assume that $M$ is an oriented manifold. Note that $S_{k}$ is a co-oriented submanifold of $J^{k}(M, \boldsymbol{R})$, whose coorientation is determined by choosing an order in the monomials $t^{\alpha}$.

Definition 2.10. Let $g: M \rightarrow \boldsymbol{R}^{n}$ be a smooth map from an oriented $m$-manifold into $\boldsymbol{R}^{n}$. Let $p \in M$ be a non-flat isolated rounding of $g$ and suppose also that $v=v\left(m_{(k)}\right)=$ $n+m$. There exists a unique $x_{0} \in \boldsymbol{R}^{n}$ such that $\left(x_{0}, p\right) \in \Sigma^{m_{(k)}}(D)$. Then, we define the index of the rounding $p$, $\operatorname{ind}_{\mathcal{R}}(g, p)$, as the oriented intersection number of the map

$$
\left(\boldsymbol{R}^{n} \times M,\left(x_{0}, p\right)\right) \rightarrow\left(J^{k}(M, \boldsymbol{R}), j_{x_{0}}^{k}(t)\right), \quad(x, t) \mapsto j^{k} d_{x}(t),
$$

and $S_{k}$ at $\left(x_{0}, p\right)$. By taking local coordinates we can assume that $M=U \subset \boldsymbol{R}^{m}$, an open subset. Then, $\operatorname{ind}_{\mathcal{R}}(g, p)$ is equal to the mapping degree of the map germ

$$
\theta_{\mathcal{R}}:\left(\boldsymbol{R}^{n} \times \boldsymbol{R}^{m},\left(x_{0}, p\right)\right) \rightarrow\left(\boldsymbol{R}^{\nu}, 0\right), \quad(x, t) \mapsto\left(\frac{\partial^{|\alpha|} d_{x}}{\partial t^{\alpha}}\right)_{1 \leq|\alpha| \leq k} .
$$

To define the index of a flattening we have to be more careful. Even when it has rank $n-1$, there are two possible vectors $v_{0} \in S^{n-1}$, such that $\left(v_{0}, t_{0}\right) \in \Sigma^{m_{(k)}}(H)$, namely $\pm v_{0}$.

DEFinition 2.11. Let $g: M \rightarrow \boldsymbol{R}^{n}$ be a smooth map from an oriented $m$-manifold into $\boldsymbol{R}^{n}$. Assume that the sphere $S^{n-1}$ is oriented. Let $p \in M$ be an isolated flattening of $g$ of rank $n-1$ and suppose also that $v=v\left(m_{(k)}\right)=n+m-1$. There is $v_{0} \in S^{-1}$ such that 
$\left(v_{0}, p\right) \in \Sigma^{m_{(k)}}(H)$. Then, we define the index of the flattening $p$, ind $\operatorname{F}_{\mathcal{F}}(g, p)$, as the oriented intersection number of the map

$$
\left(S^{n-1} \times M,\left(v_{0}, p\right)\right) \rightarrow\left(J^{k}(M, \boldsymbol{R}), j^{k} h_{v_{0}}(p)\right), \quad(v, t) \mapsto j^{k} h_{v}(t),
$$

and $S_{k}$ at $\left(v_{0}, p\right)$. Again, we can take local coordinates and assume that $M=U \subset \boldsymbol{R}^{m}$, an open subset. Then, $\operatorname{ind}_{\mathcal{F}}(g, p)$ is equal to the mapping degree of the map germ

$$
\theta_{\mathcal{F}}:\left(S^{n-1} \times \boldsymbol{R}^{m},\left(v_{0}, p\right)\right) \rightarrow\left(\boldsymbol{R}^{\nu}, 0\right), \quad(v, t) \mapsto\left(\frac{\partial^{|\alpha|} h_{v}}{\partial t^{\alpha}}\right)_{1 \leq|\alpha| \leq k} .
$$

Note that we have exactly two choices for the vector, namely $\pm v_{0}$. Thus, when $v=n+m-1$ is even, $\operatorname{ind}_{\mathcal{F}}(g, p)$ is well defined and does not depend on $v_{0}$. However, when $v=n+m-1$ is odd, we can only consider $|\operatorname{ind} \mathcal{F}(g, p)|$.

It follows that if $g: M \rightarrow \boldsymbol{R}^{n}$ is generic and $v=v\left(m_{(k)}\right)=n+m$, then all its roundings are non-flat, isolated and have index \pm 1 . When $g$ is not generic, but the rounding $p \in M$ is non-flat and isolated, we can use the Thom's transversality theorem and take a generic deformation $g_{\lambda}: M \rightarrow \boldsymbol{R}^{n}, \lambda \in(-\varepsilon, \varepsilon)$, so that the index is equal to the sum of the indices of the generic roundings that appear in $g_{\lambda}$ near $p$. Moreover, we have an analogous statement for flattenings. The following result is a consequence of Lemma 2.7.

COROLLARY 2.12. Let $g: M \rightarrow \boldsymbol{R}^{n}$ be a smooth map from an oriented smooth $m$ manifold in $\boldsymbol{R}^{n}$.

(1) If $p \in M$ is a rounding of finite multiplicity and $v\left(m_{(k)}\right)=n+m$, then $\left|\operatorname{ind}_{\mathcal{R}}(g, p)\right|$ $\leq \mu_{\mathcal{R}}(g, p)$, and $\operatorname{ind}_{\mathcal{R}}(g, p) \equiv \mu_{\mathcal{R}}(g, p)$ modulo 2 .

(2) If $p \in M$ is $a(n-1)$-rank flattening of finite multiplicity and $\nu\left(m_{(k)}\right)=n+m-1$, then $\left|\operatorname{ind}_{\mathcal{F}}(g, p)\right| \leq \mu_{\mathcal{F}}(g, p)$ and $\operatorname{ind}_{\mathcal{F}}(g, p) \equiv \mu_{\mathcal{F}}(g, p)$ modulo 2 .

As another consequence, we obtain that when the rounding is non-flat or when the flattening has rank $n-1$, then Theorem 2.9 is also true even when the maps are smooth instead of analytic.

2.4. The stereographic projection. We finish this section by showing that roundings and flattenings are related through the stereographic projection. Let $\xi: \boldsymbol{R}^{n} \rightarrow S^{n} \hookrightarrow \boldsymbol{R}^{n+1}$ denote the inverse of the stereographic projection, which is given by

$$
\xi(x)=\frac{\left(2 x,\|x\|^{2}-1\right)}{\left(\|x\|^{2}+1\right)} .
$$

It follows from the works by Romero Fuster [26, 27] and Sedykh [29] that $p \in M$ is a rounding of a smooth map $g: M \rightarrow \boldsymbol{R}^{n}$ if and only if it is a flattening of $\xi \circ g: M \rightarrow \boldsymbol{R}^{n+1}$. In fact, if $p \in M$ is an isolated non-flat rounding of $g$, then $p$ is an isolated flattening of $\xi \circ g$ of rank $n-1$. This means that there is a unique $x_{0} \in \boldsymbol{R}^{n}$ such that $\left(x_{0}, p\right) \in \Sigma^{m_{(k)}}(D)$. However, there are two possible choices for the vector $v_{0} \in S^{n}$, so that $\left(v_{0}, p\right) \in \Sigma^{m_{(k)}}(H)$ and $H$ being the height unfolding of $\xi \circ g$. We will see that there is a canonical choice of this vector $v_{0}$ in such a way that $\operatorname{ind}_{\mathcal{R}}(g, p)=\operatorname{ind}_{\mathcal{F}}(\xi \circ g, p)$. 
Given $a \in \boldsymbol{R}^{n}$ and $r>0$, we define

$$
v(a, r)=-\left(2 a,\|a\|^{2}-r^{2}-1\right), \quad u(a, r)=\frac{v(a, r)}{\|v(a, r)\|}, \quad \rho(a, r)=-\frac{\|a\|^{2}-r^{2}+1}{\|v(a, r)\|} .
$$

Then, by direct computation we have the following.

(1) If $r=\left\|x_{0}-a\right\|$, then $\left\langle\xi\left(x_{0}\right), u(a, r)\right\rangle=\rho(a, r)$. This means that $\xi$ maps the $(n-1)$-sphere in $\boldsymbol{R}^{n}$ with center $a$ and radius $r$ into the $(n-1)$-sphere in $S^{n}$ given by the intersection with the hyperplane in $\boldsymbol{R}^{n+1}$ whose equation is $\langle y, u(a, r)\rangle=\rho(a, r)$. Obviously, this is also true if we take $-u(a, r)$ and $-\rho(a, r)$.

(2) For any $x \in \boldsymbol{R}^{n}$,

$$
\langle\xi(x), u(a, r)\rangle-\rho(a, r)=\frac{2}{\|v(a, r)\|\left(\|x\|^{2}+1\right)}\left(\|x-a\|^{2}-r^{2}\right) .
$$

Thus, the choice of $u(a, r)$ and $\rho(a, r)$ is so that we obtain the height function by multiplying the distance-squared function by a positive function. The other choice of $u(a, r)$ and $\rho(a, r)$ would give a product with a negative function.

Let $p \in M$ be an isolated non-flat rounding of $g$ and let $x_{0} \in \boldsymbol{R}^{n}$ be such that $\left(x_{0}, p\right) \in$ $\Sigma^{m_{(k)}}(D)$. Then the map $\bar{u}(x)=u(x,\|x-g(p)\|)$ is a local diffeomorphism from $\left(\boldsymbol{R}^{n}, x_{0}\right)$ into $\left(S^{n}, v_{0}\right)$, where $\bar{u}\left(x_{0}\right)=v_{0}$ and so that $\left(v_{0}, p\right) \in \Sigma^{m_{(k)}}(H)$.

THEOREM 2.13. Let $p \in M$ be an isolated non-flat rounding of $g$. Then $\operatorname{ind}_{\mathcal{R}}(g, p)=$ $\operatorname{ind}_{\mathcal{F}}(\xi \circ g, p)$, where the index of the flattening is considered with respect to the vector $v_{0}$ and we choose the orientation in $S^{n}$ determined by $\bar{u}(x)$.

PROOF. The first part is an immediate consequence of the above properties. To see the second part, Note that

$$
h_{\bar{u}(x)}(t)-h_{\bar{u}(x)}(p)=\lambda(x, t)\left(d_{x}(t)-d_{x}(p)\right),
$$

for some function $\lambda(x, t)>0$, where $d_{x}(t)$ is the distance-squared function of $g$ and $h_{v}$ is the height function of $\xi \circ g$.

By taking local coordinates in $M$, we can assume that $M=U$, an open subset of $\boldsymbol{R}^{m}$. Then,

$\frac{\partial^{|\alpha|} h_{\bar{u}(x)}}{\partial t^{\alpha}}(t)=\lambda(x, t) \frac{\partial^{|\alpha|} d_{x}}{\partial t^{\alpha}}(t)+\sum_{1 \leq|\beta|<|\alpha|} \lambda_{\alpha, \beta}(x, t) \frac{\partial^{|\beta|} d_{x}}{\partial t^{\beta}}(t)+\frac{\partial^{|\alpha|} \lambda}{\partial t^{\alpha}}(x, t)\left(d_{x}(t)-d_{x}(p)\right)$, for some functions $\lambda_{\alpha, \beta}(x, t)$. Thus,

$$
\theta_{\mathcal{F}}(\xi \circ g,(\bar{u}(x), t))=\Lambda(x, t) \theta_{\mathcal{R}}(g,(x, t))+A(x, t)\left(d_{x}(t)-d_{x}(p)\right),
$$

where $\Lambda(x, t)$ is a $v \times v$ matrix whose determinant is $\lambda(x, t)^{v}>0$ and $A(x, t) \in \boldsymbol{R}^{v}$. In particular, this implies that the mapping degree of $\theta_{\mathcal{F}}$ at $\left(v_{0}, p\right)$ is equal to the mapping degree of $\theta_{\mathcal{R}}$ at $\left(x_{0}, p\right)$

3. Curves in $\boldsymbol{R}^{n}$. Let $g: I \rightarrow \boldsymbol{R}^{n}$ be a smooth curve in $\boldsymbol{R}^{n}$, where $I \subset \boldsymbol{R}$ is an interval. We have that $m=1$ and $v\left(m_{(k)}\right)=k$, so that we can consider $k$-roundings with 
$k=n+1$ and $k$-flattenings with $k=n$. In fact, if $g$ is given by $g(t)=\left(g_{1}(t), \ldots, g_{n}(t)\right)$, then

$$
F(g, t)=\left(\begin{array}{cccc}
g_{1}^{\prime}(t) & g_{2}^{\prime}(t) & \cdots & g_{n}^{\prime}(t) \\
g_{1}^{\prime \prime}(t) & g_{2}^{\prime \prime}(t) & \cdots & g_{n}^{\prime \prime}(t) \\
g_{1}^{\prime \prime \prime}(t) & g_{2}^{\prime \prime \prime}(t) & \cdots & g_{n}^{\prime \prime \prime}(t) \\
\vdots & \vdots & \ddots & \vdots \\
g_{1}^{(n)}(t) & g_{2}^{(n)}(t) & \cdots & g_{n}^{(n)}(t)
\end{array}\right)
$$

and $t \in I$ is a flattening of $g$ if and only if the determinant of the matrix $F(g, t)$ is zero at $t$. This definition agrees with the ordinary definition of flattening when $g^{\prime}(t), \ldots, g^{(n-1)}(t)$ are linearly independent at any point $t \in I$, so that $g$ has a well-defined Frenet frame and curvatures $\kappa_{1}, \ldots, \kappa_{n-1}$. In this case it is obvious that $t \in I$ is a flattening if and only if $\kappa_{n-1}(t)=0$.

We next set

$$
R(g, t)=\left(\begin{array}{ccccc}
g_{1}^{\prime}(t) & g_{2}^{\prime}(t) & \cdots & g_{n}^{\prime}(t) & 0 \\
g_{1}^{\prime \prime}(t) & g_{2}^{\prime \prime}(t) & \cdots & g_{n}^{\prime \prime}(t) & \varphi_{1}(t) \\
g_{1}^{\prime \prime \prime}(t) & g_{2}^{\prime \prime \prime}(t) & \cdots & g_{n}^{\prime \prime \prime}(t) & \varphi_{2}(t) \\
\vdots & \vdots & \ddots & \vdots & \vdots \\
g_{1}^{(n+1)}(t) & g_{2}^{(n+1)}(t) & \cdots & g_{n}^{(n+1)}(t) & \varphi_{n}(t)
\end{array}\right),
$$

where $\varphi_{k}$ satisfy the following formula:

$$
\begin{gathered}
\varphi_{1}(t)=g_{1}^{\prime}(t)^{2}+\cdots+g_{n}^{\prime}(t)^{2} \\
\varphi_{k}(t)=\sum_{i=1}^{n} g_{i}^{(k)}(t) g_{i}^{\prime}(t)+\varphi_{k-1}^{\prime}(t), \quad k=2, \ldots, n .
\end{gathered}
$$

Then $t \in I$ is a rounding of $g$ if and only if the determinant of the matrix $R(g, t)$ is zero at $t$. Again, in the regular case, we can translate this condition in terms of the Frenet frame $\mathbf{e}_{1}, \ldots, \mathbf{e}_{n}$ and the curvatures $\kappa_{1}, \ldots, \kappa_{n-1}$.

Definition 3.1. Let $g: I \rightarrow \boldsymbol{R}^{n}$ be a smooth curve such that $g^{\prime}(t), \ldots, g^{(n-1)}(t)$ are linearly independent at any point $t \in I$, and assume that it is parameterized by arc length. At a point $t \in I$, where $\kappa_{n-1}(t) \neq 0$, the center of the osculating hypersphere is the only point $\mu(t) \in \boldsymbol{R}^{n}$ defined by the condition $(\mu(t), t) \in \Sigma^{1_{(n)}}(D)$.

The proof of the following result can be found in [28].

Proposition 3.2. Let $g: I \rightarrow \boldsymbol{R}^{n}$ be as in Definition 3.1. Then,

$$
\mu=g+\mu_{1} \mathbf{e}_{1}+\cdots+\mu_{n} \mathbf{e}_{n},
$$

where $\mu_{1}=0, \mu_{2}=1 / \kappa_{1}, \mu_{3}=\mu_{2}^{\prime} / \kappa_{2}$ and

$$
\mu_{k+1}=\left(\mu_{k}^{\prime}+\kappa_{k-1} \mu_{k-1}\right) / \kappa_{k} \text { for } k=3, \ldots, n-1 .
$$

Moreover, $t$ is a rounding if and only if

$$
\mu_{n}^{\prime}(t)+\kappa_{n-1}(t) \mu_{n-1}(t)=0 .
$$




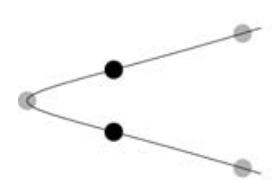

$\lambda>0$

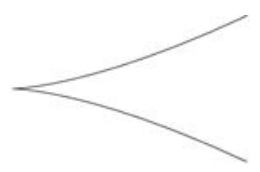

$\lambda=0$

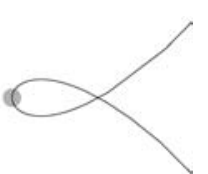

$\lambda<0$

FIGURE 1

EXAMPLE 3.3. If $n=2$, then $t \in I$ is a rounding if and only if $\mu_{2}^{\prime}(t)=0$, where $\mu_{2}=1 / \kappa$. Obviously, this is equivalent to $\kappa^{\prime}(t)=0$, which is the ordinary definition of a vertex of a regular plane curve. In fact, in this case it is possible to show that

$$
\operatorname{det} R(g, t)=-\kappa^{\prime}(t)\left\|g^{\prime}(t)\right\|^{5},
$$

which implies that $t$ is a rounding if and only if it is a vertex, even when $\kappa=0$.

The multiplicity of a flattening or a rounding is determined by the first non-zero term in the Taylor expansion of $\operatorname{det} F(g, t)$ or $\operatorname{det} R(g, t)$, respectively. For instance, if we suppose that $t_{0}=0$ is a flattening and $\operatorname{det} F(g, t)=a_{k} t^{k}+\cdots$, with $a_{k} \neq 0$, then $\mu_{\mathcal{F}}(g, 0)=k$.

EXAMPLE 3.4. Let $g: \boldsymbol{R} \rightarrow \boldsymbol{R}^{2}$ be the plane curve defined by $t \mapsto\left(t^{2}, t^{3}\right)$. It is easy to see that this curve has a flattening and a rounding at $t=0$ with multiplicities $\mu_{\mathcal{F}}(g, 0)=2$ and $\mu_{\mathcal{R}}(g, 0)=3$.

We consider now the deformation $g_{\lambda}$ defined by $g_{\lambda}(t)=\left(t^{2},\left(\lambda+t^{2}\right) t\right)$. The curvature and its derivative are given by the following formulas:

$$
\kappa_{\lambda}(t)=\frac{2\left(3 t^{2}-\lambda\right)}{\left(4 t^{2}+\left(\lambda+3 t^{2}\right)^{2}\right)^{3 / 2}} \quad \text { and } \quad \kappa_{\lambda}^{\prime}(t)=\frac{24 t\left(-9 t^{4}+(3 \lambda-1) t^{2}+\lambda+2 \lambda^{2}\right)}{\left(4 t^{2}+\left(\lambda+3 t^{2}\right)^{2}\right)^{5 / 2}} .
$$

Thus, $g_{\lambda}$ has two flattenings and three vertices near 0 if $\lambda$ is a small positive number and $g_{\lambda}$ has no flattening points and one vertex near 0 if $\lambda$ is a small negative number (see Figure 1 ).

EXAMPLE 3.5. Let $e_{0}, e_{1}, e_{2}$ be non-negative integers. We consider the plane curve $g: \boldsymbol{R} \rightarrow \boldsymbol{R}^{2}$ defined by

$$
g(t)=\left(t^{e_{0}}, t^{e_{0}+e_{1}}+a t^{e_{0}+e_{1}+e_{2}}\right) .
$$

Then, by elementary computation, we have

$$
\begin{aligned}
\operatorname{det} F(g, t)= & e_{0} e_{1}\left(e_{0}+e_{1}\right) t^{2 e_{0}+e_{1}-3}+\cdots, \\
\operatorname{det} R(g, t)= & e_{0}^{3} e_{1}\left(e_{0}+e_{1}\right)\left(e_{0}-e_{1}\right) t^{4 e_{0}+e_{1}-6}+e_{0} e_{1}\left(e_{0}+e_{1}\right)^{3}\left(e_{0}+2 e_{1}\right) t^{4 e_{0}+3 e_{1}-6} \\
& +a e_{0}^{3}\left(e_{0}-e_{1}-e_{2}\right)\left(e_{1}+e_{2}\right)\left(e_{0}+e_{1}+e_{2}\right)\left(e_{0}+2 e_{1}\right) t^{4 e_{0}+e_{1}+e_{2}-6}+\cdots .
\end{aligned}
$$

In this case, the multiplicities are given by $\mu_{\mathcal{F}}(g, 0)=2 e_{0}+e_{1}-3$ and

$$
\mu_{\mathcal{R}}(g, 0)= \begin{cases}4 e_{0}+e_{1}-6 & \text { if } e_{0} \neq e_{1}, \\ 7 e_{0}-6 & \text { if } e_{0}=e_{1}, 2 e_{1}<e_{2}, \\ 7 e_{0}-6 & \text { if } e_{0}=e_{1}, 2 e_{1}=e_{2}, a \neq 1, \\ 5 e_{0}+e_{2}-6 & \text { if } e_{0}=e_{1}, 2 e_{1}>e_{2}, a \neq 0\end{cases}
$$


The mapping degree of det $F(g, t)$ or $\operatorname{det} R(g, t)$ is equal, up to the sign, to the index of an isolated flattening or rounding, respectively, whenever they are defined. This follows from the proof of Lemma 2.7. However, we have to be careful if we want to take into account the sign of the index. We only show the following result for roundings, where the sign makes sense in any dimension.

Proposition 3.6. Let $g: I \rightarrow \boldsymbol{R}^{n}$ be a smooth curve and let $t_{0} \in I$ be an isolated non-flat rounding. Then, the index $\operatorname{ind}_{\mathcal{R}}\left(g, t_{0}\right)$ is equal to the mapping degree of $-\operatorname{det} R(g, t)$ at $t_{0}$.

Proof. The index $\operatorname{ind}_{\mathcal{R}}\left(g, t_{0}\right)$ is the mapping degree of $\theta_{\mathcal{R}}:\left(\boldsymbol{R}^{n+1},\left(x_{0}, t_{0}\right)\right) \rightarrow$ $\left(\boldsymbol{R}^{n+1}, 0\right)$ defined by

$$
\theta_{\mathcal{R}}(x, t)=\left(\left\langle g^{(i)}, x-g\right\rangle-\varphi_{i-1}\right)_{i=1}^{n+1},
$$

where $x_{0} \in \boldsymbol{R}^{n}$ is the only point such that $\theta_{\mathcal{R}}\left(x_{0}, t_{0}\right)=0$.

Since we suppose that $t_{0}$ is a non-flat rounding, we have that $g^{\prime}, \ldots, g^{(n+1)}$ have rank $n$ at $t_{0}$. We assume, for instance, that $g^{\prime}, \ldots, g^{(n)}$ are linearly independent (the other cases should be similar). Then, we can take the following change of coordinates:

$$
\bar{x}_{i}=\left\langle g^{(i)}, x-g\right\rangle-\varphi_{i-1}, \quad i=1, \ldots, n .
$$

This coordinate change transforms $\theta_{\mathcal{R}}$ into

$$
\bar{\theta}_{\mathcal{R}}(\bar{x}, t)=\left(\bar{x},\left\langle g^{(n+1)}, \phi(\bar{x}, t)-g\right\rangle-\varphi_{n}\right),
$$

where $\phi(\bar{x}, t)$ denotes the inverse of the coordinate change. In particular, this implies that

$$
\operatorname{ind}_{\mathcal{R}}\left(g, t_{0}\right)=\operatorname{sign}\left(\operatorname{det}\left(g^{\prime}, \ldots, g^{(n)}\right)\right) \operatorname{deg}\left(\bar{\theta}_{\mathcal{R}},\left(0, t_{0}\right)\right) .
$$

However, we now have that the mapping degree of $\bar{\theta}_{\mathcal{R}}$ at $\left(0, t_{0}\right)$ is equal to the local degree of $\left\langle g^{(n+1)}, \phi(0, t)-g\right\rangle-\varphi_{n}$ at $t_{0}$ (since $\bar{\theta}_{\mathcal{R}}$ is an unfolding of this function). Thus, it only remains to show that

$$
\left.\operatorname{det} R(g, t)=-\operatorname{det}\left(g^{\prime}, \ldots, g^{(n)}\right)\left(\left\langle g^{(n+1)}, \phi(0, t)-g\right\rangle-\varphi_{n}\right)\right) .
$$

Let us denote by $R_{i}$ the $(n+1, i)$ minor of $R$; that is,

$$
R_{i}=\left|\begin{array}{cccccc}
g_{1}^{\prime} & \cdots & \widehat{g_{i}^{\prime}} & \cdots & g_{n}^{\prime} & 0 \\
\vdots & & \vdots & & \vdots & \vdots \\
g_{1}^{(n)} & \cdots & \widehat{g_{i}^{(n)}} & \cdots & g_{n}^{(n)} & \varphi_{n-1}
\end{array}\right| .
$$

It follows that

$$
\operatorname{det} R(g, t)=\varphi_{n} \operatorname{det}\left(g^{\prime}, \ldots, g^{(n)}\right)+\sum_{i=1}^{n}(-1)^{n+i+1} g_{i}^{(n+1)} R_{i} .
$$

On the other hand, $\phi(0, t)-g$ is determined by the linear system

$$
\left\langle g^{(i)}, \phi(0, t)-g\right\rangle=\varphi_{i-1}, \quad i=1, \ldots, n .
$$


Thus, by the Cramer rule, we have that

$$
\phi_{i}(0, t)-g_{i}=(-1)^{n+i} \frac{R_{i}}{\operatorname{det}\left(g^{\prime}, \ldots, g^{(n)}\right)} .
$$

In particular,

$$
\operatorname{det} R(g, t)=\varphi_{n} \operatorname{det}\left(g^{\prime}, \ldots, g^{(n)}\right)-\sum_{i=1}^{n} g_{i}^{(n+1)}\left(\phi_{i}(0, t)-g_{i}\right) \operatorname{det}\left(g^{\prime}, \ldots, g^{(n)}\right),
$$

which gives the desired result.

4. Surfaces in $\boldsymbol{R}^{3}$. In this section, we suppose that $g: U \subset \boldsymbol{R}^{2} \rightarrow \boldsymbol{R}^{3}$ is a smooth map given by $g(u, v)=\left(g_{1}(u, v), g_{2}(u, v), g_{3}(u, v)\right)$ which defines a surface in $\boldsymbol{R}^{3}$ (possibly with singularities). Then the first and the second fundamental forms for $g$ are given by

$$
\mathrm{I}=E d u^{2}+2 F d u d v+G d v^{2}, \quad \mathrm{II}=L d u^{2}+2 M d u d v+N d v^{2}
$$

where

$$
\begin{gathered}
E=\left\langle g_{u}, g_{u}\right\rangle, \quad F=\left\langle g_{u}, g_{v}\right\rangle, \quad G=\left\langle g_{v}, g_{v}\right\rangle \\
L=\frac{\operatorname{det}\left(g_{u}, g_{v}, g_{u u}\right)}{\sqrt{E G-F^{2}}}, \quad M=\frac{\operatorname{det}\left(g_{u}, g_{v}, g_{u v}\right)}{\sqrt{E G-F^{2}}}, \quad N=\frac{\operatorname{det}\left(g_{u}, g_{v}, g_{v v}\right)}{\sqrt{E G-F^{2}}},
\end{gathered}
$$

and subscripts denote partial derivatives. Naturally, $L, M, N$ are only defined if the denominator does not vanish; that is, at the regular points of $g$. Umbilics are regular points of the surface so that the second fundamental form is proportional to the first. Thus, we say that $(u, v)$ is an umbilic of $g$ if the rank of the matrix

$$
\left(\begin{array}{lll}
E & F & G \\
L & M & N
\end{array}\right)
$$

is less than 2. Moreover, it is well-known that umbilic points of a regular surface correspond to points where the two principal curvatures (or eigenvalues of the second fundamental form) are equal.

In order to include the case where $g$ may have singularities, we can define a new matrix

$$
U(g,(u, v))=\left(\begin{array}{ccc}
E & F & G \\
L^{\prime} & M^{\prime} & N^{\prime}
\end{array}\right)
$$

where $L^{\prime}=\operatorname{det}\left(g_{u}, g_{v}, g_{u u}\right), M^{\prime}=\operatorname{det}\left(g_{u}, g_{v}, g_{u v}\right), N^{\prime}=\operatorname{det}\left(g_{u}, g_{v}, g_{v v}\right)$. This matrix always makes sense. We observe that the rank of this matrix is not maximal either at an umbilic or at a singular point of $g$.

4.1. 2-roundings of $g$. Now we look at 2-roundings (rounding, for short) of $g$. It makes sense to look at $k$-roundings of $g$, with $k=2$. A rounding is a point where the rank of 
the matrix

$$
R(g,(u, v))=\left(\begin{array}{cccc}
g_{1 u} & g_{2 u} & g_{3 u} & 0 \\
g_{1 v} & g_{2 v} & g_{3 v} & 0 \\
g_{1 u u} & g_{2 u u} & g_{3 u u} & E \\
g_{1 u v} & g_{2 u v} & g_{3 u v} & F \\
g_{1 v v} & g_{2 v v} & g_{3 v v} & G
\end{array}\right)
$$

is less than four.

The following result is well-known (see Porteous [24]).

PROPOSITION 4.1. A regular point of a smooth map $g: U \subset \boldsymbol{R}^{2} \rightarrow \boldsymbol{R}^{3}$ is a rounding if and only if it is an umbilic. Moreover, it is non-flat if and only if the principal curvature is not zero.

Now, we see that in the singular case, a rounding corresponds to a singular point which is not of Whitney umbrella type. Remember that a singular point is a Whitney umbrella if the map is generic in the Thom-Boardman sense (that is, its jet extension is transverse to the Boardman strata).

Proposition 4.2. A singular point of a smooth map $g: U \subset \boldsymbol{R}^{2} \rightarrow \boldsymbol{R}^{3}$ is a rounding if and only if it is not a Whitney umbrella.

PROOF. If the map $g$ has rank zero at $\left(u_{0}, v_{0}\right)$, it follows that it is always a rounding, but it is not a Whitney umbrella (since the 1-jet extension of $g$ is not transverse to $\Sigma^{2}$ ).

Suppose now that $g$ has rank one at $\left(u_{0}, v_{0}\right)$. Again we may assume that $\left(u_{0}, v_{0}\right)=(0,0)$ and $g$ is given by

$$
g(u, v)=\left(u, g_{2}(u, v), g_{3}(u, v)\right) .
$$

Then our matrix $R(g,(u, v))$ can be written in the following form:

$$
\left(\begin{array}{cccc}
1 & g_{2 u} & g_{3 u} & 0 \\
0 & g_{2 v} & g_{3 v} & 0 \\
0 & g_{2 u u} & g_{3 u u} & E \\
0 & g_{2 u v} & g_{3 u v} & F \\
0 & g_{2 v v} & g_{3 v v} & G
\end{array}\right) \quad\left\{\begin{array}{l}
E=1+g_{2 u}^{2}+g_{3}^{2}, \\
F=g_{2 u} g_{2 v}+g_{3 u} g_{3 v}, \\
G=g_{2 v}^{2}+g_{3 v}^{2} .
\end{array}\right.
$$

On the other hand, the map $g$ defines a Whitney umbrella if and only if its 2-jet section is transverse to the $\Sigma^{1}$ strata. This holds if and only if

$$
\left|\begin{array}{ll}
g_{2 u v} & g_{3 u v} \\
g_{2 v v} & g_{3 v v}
\end{array}\right| \neq 0 \text { at } 0
$$

This condition is equivalent to $\operatorname{rank} R(g, 0)=4$.

In the last part of this section, we study the index of a non-flat isolated rounding. From Definition 2.10 we have that if $p \in U$ is a non-flat isolated rounding of $g: U \subset \boldsymbol{R}^{2} \rightarrow \boldsymbol{R}^{3}$, then there exists a unique $x_{0} \in \boldsymbol{R}^{3}$ such that $\left(x_{0}, p\right) \in \Sigma^{2,2}(D)$. Then $\operatorname{ind}_{\mathcal{R}}(g, p)$ is equal to the mapping degree of the smooth map germ $\theta:\left(\boldsymbol{R}^{5},\left(x_{0}, p\right)\right) \rightarrow\left(\boldsymbol{R}^{5}, 0\right)$ whose components 
$\theta_{i}, i=1, \ldots, 5$, are given by the following matrix equation:

$$
\left(\begin{array}{l}
\theta_{1} \\
\theta_{2} \\
\theta_{3} \\
\theta_{4} \\
\theta_{5}
\end{array}\right)=\left(\begin{array}{ccc}
g_{1 u} & g_{2 u} & g_{3 u} \\
g_{1 v} & g_{2 v} & g_{3 v} \\
g_{1 u u} & g_{2 u u} & g_{3 u u} \\
g_{1 u v} & g_{2 u v} & g_{3 u v} \\
g_{1 v v} & g_{2 v v} & g_{3 v v}
\end{array}\right)\left(\begin{array}{l}
x \\
y \\
z
\end{array}\right)+\left(\begin{array}{l}
0 \\
0 \\
E \\
F \\
G
\end{array}\right)
$$

Let us denote by $P_{u}, P_{v}, P_{u u}, P_{u v}, P_{v v}$ the five maximal minors of the matrix $R(g,(u, v))$. That is,

$$
P_{u}=\left|\begin{array}{ll}
g_{v} & 0 \\
g_{u u} & E \\
g_{u v} & F \\
g_{v v} & G
\end{array}\right|, \quad P_{v}=\left|\begin{array}{ll}
g_{u} & 0 \\
g_{u u} & E \\
g_{u v} & F \\
g_{v v} & G
\end{array}\right|, \quad P_{u u}=\left|\begin{array}{cc}
g_{u} & 0 \\
g_{v} & 0 \\
g_{u v} & F \\
g_{v v} & G
\end{array}\right|, \quad P_{u v}=\left|\begin{array}{cc}
g_{u} & 0 \\
g_{v} & 0 \\
g_{u u} & E \\
g_{v v} & G
\end{array}\right|, \quad P_{v v}=\left|\begin{array}{cc}
g_{u} & 0 \\
g_{v} & 0 \\
g_{u u} & E \\
g_{u v} & F
\end{array}\right| .
$$

If we look at the proof of Lemma 2.7, we see that when the rounding is non-flat, then

$$
C^{\infty}\left(\boldsymbol{R}^{5},\left(x_{0}, p\right)\right) /\left\langle\theta_{1}, \ldots, \theta_{5}\right\rangle \cong C^{\infty}\left(\boldsymbol{R}^{2}, p\right) /\left\langle P_{u}, P_{v}, P_{u u}, P_{u v}, P_{v v}\right\rangle .
$$

This implies that we can choose a pair of elements $P, Q \in\left\{P_{u}, P_{v}, P_{u u}, P_{u v}, P_{v v}\right\}$ which generate the ideal $\mathcal{R}(g, p)$. Moreover, we have that the mapping degree of $\theta$ is equal, up to sign, to the mapping degree of $(P, Q):\left(\boldsymbol{R}^{2}, p\right) \rightarrow\left(\boldsymbol{R}^{2}, 0\right)$.

The relations among $P_{u}, P_{v}, P_{u u}, P_{u v}, P_{v v}$ are given in the following form:

$$
\begin{gathered}
g_{i u} P_{u}-g_{i v} P_{v}+g_{i u u} P_{u u}-g_{i u v} P_{u v}+g_{i v v} P_{v v}=0, \quad i=1,2,3, \\
E P_{u u}-F P_{u v}+G P_{v v}=0 .
\end{gathered}
$$

4.2. Principal directions. We have another index at an isolated rounding, which is associated with the principal directions of $g$. Suppose that $(u, v)$ is a regular point which is not umbilic. Then the principal directions of $g$ at $(u, v)$ are defined as the directions determined by the eigenvectors of the second fundamental form at $(u, v)$. Then we find that the principal directions are given by the equation

$$
\left|\begin{array}{ccccc}
g_{1 u} & g_{2 u} & g_{3 u} & 0 & 0 \\
g_{1 v} & g_{2 v} & g_{3 v} & 0 & 0 \\
g_{1_{u u}} & g_{2 u u} & g_{3 u u} & E & d v^{2} \\
g_{1_{u v}} & g_{2 u v} & g_{3 u v} & F & -d u d v \\
g_{1 v v} & g_{2 v v} & g_{3 v v} & G & d u^{2}
\end{array}\right|=P_{u u} d v^{2}+P_{u v} d u d v+P_{v v} d u^{2}=0
$$

Thus, the principal directions define a pair of orthogonal line fields in the surface, which are singular either at an umbilic or at a singular point of $g$. If $p \in U$ is an isolated umbilic or an isolated singular point of $g$, we denote by $\operatorname{ind}_{\mathcal{P}}(g, p)$ the index at $p$ associated with any of these line fields. This is a half-integer and is equal to the number of turns of the line field when we run through a small circle in $U$ centered at $p$.

The classification of generic umbilics goes back to Darboux [5]. He found that there are three types; namely, the lemon or $D_{1}$, the monstar or $D_{2}$ and the star or $D_{3}$. Moreover, he gave a description for the configuration of the curvature lines (integral curves of the principal 


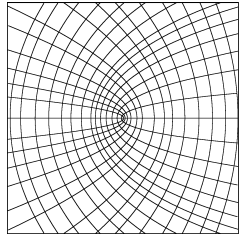

$D_{1}$

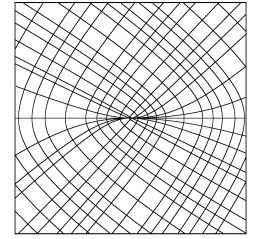

$\mathrm{D}_{2}$

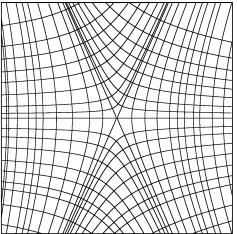

$D_{3}$

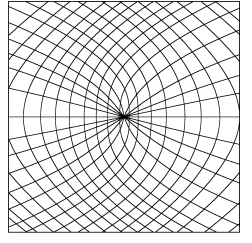

$W$

FIGURE 2.

directions) in each case. In fact, it was shown by Gutierrez and Sotomayor [10] (see also the work by Bruce and Fidal [3]) that $g$ is principally structurally stable at an umbilic if and only if it is one of the Darbouxian umbilics. By looking at these configurations, it is easy to compute the index ind $\mathcal{P}(g, p): D_{1}$ and $D_{2}$ have index $1 / 2$, while $D_{3}$ has $-1 / 2$.

The configuration of principal lines at a generic singular point can be found in [7] (this is a corrected version of some erroneous results appearing in [11]). It follows that a singular point of $g$ is principally structurally stable if and only if it is a Whitney umbrella. Moreover, from the description of principal lines at a Whitney umbrella we deduce that the index ind $_{\mathcal{P}}(g, p)$ is $1 / 2$.

In Figure 2, we present drawings for the configuration of principal lines at Darbouxian umbilics $D_{1}, D_{2}, D_{3}$ and at a Whitney umbrella $W$, obtained with the Superficies program [21]. As a consequence, if the map $g$ is not generic, we can take a generic deformation $g_{\lambda}$ and the index $\operatorname{ind}_{\mathcal{P}}(g, p)$ is equal to $\left(D_{1}+D_{2}-D_{3}+W\right) / 2$, where $D_{1}, D_{2}, D_{3}, W$ denote the number of umbilics of each type or Whitney umbrella that appear in $g_{\lambda}$ near $p$, for $\lambda \neq 0$ small enough.

Moreover, the differential equation for principal lines can be seen as a particular case of a positive quadratic differential (PQD) form in the sense of [9]. In local coordinates $(u, v)$, a PQD form is given by

$$
\omega=A(u, v) d v^{2}+B(u, v) d u d v+C(u, v) d u^{2},
$$

where $A, B, C$ are smooth functions such that $B^{2}-4 A C \geq 0$ and $B^{2}-4 A C=0$ if and only if $A=B=C=0$. The points where $A=B=C=0$ are called singular points of $\omega$. Associated with $\omega$ we have a pair of transversal line fields corresponding to the roots of $\omega$, which become singular precisely at the singular points of $\omega$. Thus, if $p$ is an isolated singular point of $\omega$, we can consider the index ind $(\omega, p)$ associated with any of these line fields.

The following lemma, whose proof can be found in [13, Part 2, VIII, 2.3], shows that the index of an isolated singular point of a PQD form is related to the mapping degree of the coefficients.

LEMMA 4.3. Let $p$ be an isolated singular point of the $P Q D$ form $\omega=A(u, v) d v^{2}+$ $B(u, v) d u d v+C(u, v) d u^{2}$. Then,

$$
\operatorname{ind}(\omega, p)=-\frac{1}{2} \operatorname{deg}((A, B), p)=-\frac{1}{2} \operatorname{deg}((B, C), p),
$$


where $\operatorname{deg}((A, B), p)$ and $\operatorname{deg}((B, C), p)$ denote the mapping degrees of the maps $(A, B)$ and $(B, C)$, respectively, at $p$.

As an immediate consequence, we get the following result, which allows us to compute the index of the principal foliation in terms of the mapping degree of the coefficients $P_{u u}, P_{u v}, P_{v v}$.

COROLlary 4.4. Let $g: U \subset \boldsymbol{R}^{2} \rightarrow \boldsymbol{R}^{3}$ be a smooth map and let $p \in U$ be either an isolated umbilic or an isolated singular point of $g$. Then,

$$
\begin{aligned}
\operatorname{ind}_{\mathcal{P}}(g, p) & =-\frac{1}{2} \operatorname{deg}\left\{\left(P_{u u}, P_{u v}\right) ;\left(\boldsymbol{R}^{2}, p\right) \rightarrow\left(\boldsymbol{R}^{2}, 0\right)\right\} \\
& =-\frac{1}{2} \operatorname{deg}\left\{\left(P_{u v}, P_{v v}\right) ;\left(\boldsymbol{R}^{2}, p\right) \rightarrow\left(\boldsymbol{R}^{2}, 0\right)\right\} .
\end{aligned}
$$

4.3. Indices of roundings. We apply Corollary 4.4 to find relations between the index defined by principal directions $\operatorname{ind}_{\mathcal{P}}(g, p)$ and the index $\operatorname{ind}_{\mathcal{R}}(g, p)$. However, we have to distinguish cases depending on the rank of $g$ at $p$.

4.3.1. Rank two case. We start by considering the case where $p$ is a regular point.

THEOREM 4.5. Let $g: U \subset \boldsymbol{R}^{2} \rightarrow \boldsymbol{R}^{3}$ be a smooth map and let $p \in U$ be a non-flat isolated umbilic of $g$ with principal curvature $k \neq 0$. Then,

$$
\operatorname{ind}_{\mathcal{P}}(g, p)=\frac{1}{2} \operatorname{sign}(k) \operatorname{ind}_{\mathcal{R}}(g, p) .
$$

Proof. We will assume for simplicity that $p=0$ is a non-flat umbilic and that $g$ is given by the Monge normal form $g(u, v)=(u, v, w(u, v))$. The matrix $R(g,(u, v))$ is equal to

$$
\left(\begin{array}{cccc}
1 & 0 & w_{u} & 0 \\
0 & 1 & w_{v} & 0 \\
0 & 0 & w_{u u} & 1+w_{u}^{2} \\
0 & 0 & w_{u v} & w_{u} w_{v} \\
0 & 0 & w_{v v} & 1+w_{v}^{2}
\end{array}\right)
$$

and

$$
P_{u v}=w_{u u}\left(1+w_{v}^{2}\right)-w_{v v}\left(1+w_{u}^{2}\right), \quad P_{v v}=w_{u u} w_{u} w_{v}-w_{u v}\left(1+w_{u}^{2}\right) .
$$

On the other hand, the index $\operatorname{ind}_{\mathcal{R}}(g, p)$ is equal to the mapping degree of the map germ $\theta$ given by

$$
\left(\begin{array}{l}
\theta_{1} \\
\theta_{2} \\
\theta_{3} \\
\theta_{4} \\
\theta_{5}
\end{array}\right)=\left(\begin{array}{lll}
1 & 0 & w_{u} \\
0 & 1 & w_{v} \\
0 & 0 & w_{u u} \\
0 & 0 & w_{u v} \\
0 & 0 & w_{v v}
\end{array}\right)\left(\begin{array}{l}
x \\
y \\
z
\end{array}\right)+\left(\begin{array}{c}
0 \\
0 \\
1+w_{u}^{2} \\
w_{u} w_{v} \\
1+w_{v}^{2}
\end{array}\right)
$$

at the point $P=\left(x_{0}, y_{0}, z_{0}, 0,0\right)$ such that $\theta\left(x_{0}, y_{0}, z_{0}, 0,0\right)=0$. 
We can define new coordinates $\tilde{x}, \tilde{y}, \tilde{z}$ by

$$
\left(\begin{array}{c}
\tilde{x} \\
\tilde{y} \\
\tilde{z}
\end{array}\right)=\left(\begin{array}{ccc}
1 & 0 & w_{u} \\
0 & 1 & w_{v} \\
0 & 0 & w_{u u}
\end{array}\right)\left(\begin{array}{l}
x \\
y \\
z
\end{array}\right)+\left(\begin{array}{c}
0 \\
0 \\
1+w_{u}^{2}
\end{array}\right)
$$

Note that $k \neq 0$ implies that $w_{u u} \neq 0$. Moreover, $\operatorname{sign}(k)=\operatorname{sign}\left(w_{u u}\right)$. Then, it follows that $\operatorname{deg} \theta=\operatorname{sign}(k) \operatorname{deg} \tilde{\theta}$, where $\tilde{\theta}$ is the composite of $\theta$ with such coordinate change. A simple computation gives that

$$
\tilde{\theta}(\tilde{x}, \tilde{y}, \tilde{z}, u, v)=\left(\tilde{x}, \tilde{y}, \tilde{z}, \frac{w_{u v} \tilde{z}+P_{v v}}{w_{u u}}, \frac{w_{v v} \tilde{z}+P_{u v}}{w_{u u}}\right) .
$$

In particular, we get that

$\operatorname{deg} \tilde{\theta}=\operatorname{deg}\left\{\left(\frac{P_{v v}}{w_{u u}}, \frac{P_{u v}}{w_{u u}}\right) ;\left(\boldsymbol{R}^{2}, 0\right) \rightarrow\left(\boldsymbol{R}^{2}, 0\right)\right\}=-\operatorname{deg}\left\{\left(P_{u v}, P_{v v}\right) ;\left(\boldsymbol{R}^{2}, 0\right) \rightarrow\left(\boldsymbol{R}^{2}, 0\right)\right\}$.

COROLlary 4.6. Let $g: U \subset \boldsymbol{R}^{2} \rightarrow \boldsymbol{R}^{3}$ be a smooth map and let $p \in U$ be a non-flat isolated (either regular or singular) rounding of $g$ and let $g_{\lambda}: U \rightarrow \boldsymbol{R}^{3}$ be a generic deformation of $g$, with $\lambda \in(-\varepsilon, \varepsilon)$. Then,

$$
\operatorname{ind}_{\mathcal{R}}(g, p)=D_{1}^{+}-D_{1}^{-}+D_{2}^{+}-D_{2}^{-}-D_{3}^{+}+D_{3}^{-},
$$

where $D_{i}^{+}, D_{i}^{-}$denote the number of umbilics of type $D_{i}$ with positive or negative principal curvature, respectively, that appear in $g_{\lambda}$ near $p$.

REMARK 4.7. The classical Carathéodory conjecture states that every smooth convex embedding of a 2-sphere in $\boldsymbol{R}^{3}$ must have at least two umbilics. This conjecture has a stronger local version, known as the Loewner conjecture, which states that the $\operatorname{index}_{\operatorname{ind}} \operatorname{P}_{\mathcal{P}}(g, p)$ at any isolated umbilic $g$ of a smooth regular surface $g: U \rightarrow \boldsymbol{R}^{3}$ is always $\leq 1$. Since the sum of the indices of the umbilics of a compact immersed surface is equal to its Euler-Poincaré characteristic (according to the Poincaré-Hopf formula) it follows that the Loewner conjecture implies the Carathéodory conjecture, not only for a convex embedding of a 2 -sphere, but for any immersion (non necessarily convex). The Loewner conjecture is known to be true in the analytic case, although there is much controversy about the correct proof (see $[14,31]$ ).

It would be interesting to see if $\operatorname{ind}_{\mathcal{P}}(g, p) \leq 1$ is true or not in the rank one case and provided that $g$ is analytic. If this is true, we should obtain an extension of the Carathéodory conjecture for surfaces with rank one singularities in $\boldsymbol{R}^{3}$.

In the rank 0 case it is known that this conjecture is false. In fact, it is not difficult to construct a surface with an isolated umbilic of index two. Here we present an example which is inspired by the example given in [2]. Consider the map $g: \boldsymbol{R}^{2} \rightarrow \boldsymbol{R}^{3}$ given by $g(u, v)=(1 / 3)\left(-u^{3}-3 u v^{2}, 3 u^{2} v-v^{3}, 3 u^{4}-3 v^{4}\right)$. In Figure 3 we compute the principal lines of this surface and show that it has an isolated umbilic of index two at the origin. 


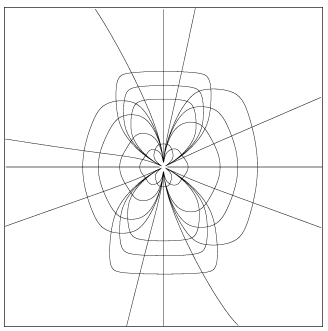

FIGURE 3.

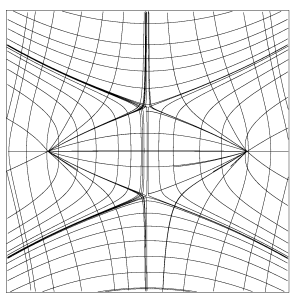

$\lambda>0$

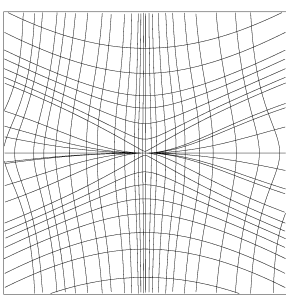

$\lambda=0$

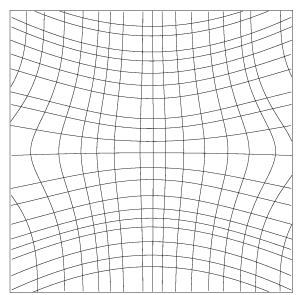

$\lambda<0$

FIGURE 4

4.3.2. Rank one case. In the rank one case, the formulas of Corollary 4.4 are still true. However, we have to be careful, because in this case, the $\operatorname{index} \operatorname{ind}_{\mathcal{P}}(g, p)$ not only counts the number of umbilics that appear in a generic deformation, but this also includes the number of Whitney umbrellas.

EXAMPLE 4.8. Let us consider the smooth family of surfaces $g_{\lambda}: \boldsymbol{R}^{2} \rightarrow \boldsymbol{R}^{3}$ defined by

$$
g_{\lambda}(u, v)=\left(u, v^{2}, v\left(\lambda+v^{2}-u^{2}\right)\right) .
$$

For $\lambda=0, g_{0}$ has an isolated singular point of rank one at 0 . For $\lambda>0, g_{\lambda}$ is generic and has two Whitney umbrellas and two umbilics of type $D_{3}$ near 0 . For $\lambda<0, g_{\lambda}$ is regular and has no umbilics. Thus, it follows that the $\operatorname{index}_{\operatorname{ind}}(g, p)$ is equal to zero. The configuration of the curvature lines near 0 is shown in Figure 4 in the three cases.

In the rank one case, we can also obtain a formula to compute the index $\operatorname{ind}_{\mathcal{R}}(g, p)$ provided that the rounding is non-flat and isolated. However, it is not clear at all which is the relation between this index and the index of the principal foliation $\operatorname{ind}_{\mathcal{P}}(g, p)$.

Assume that $p=0$ and that $g$ is given in the form $g(u, v)=\left(u, g_{2}(u, v), g_{3}(u, v)\right)$, where $g_{2}, g_{3} \in\langle u, v\rangle^{2}$. We have seen that $g$ has a rounding at 0 if and only if it is not a 
Whitney umbrella and it is non-flat if and only if the following matrix has rank two at 0 :

$$
\left(\begin{array}{cc}
g_{2 v} & g_{3 v} \\
g_{2 u u} & g_{3 u u} \\
g_{2 u v} & g_{3 u v} \\
g_{2 v v} & g_{3 v v}
\end{array}\right) .
$$

Let us denote the maximal minors of this matrix by

$$
\begin{array}{ll}
D_{12}=\left|\begin{array}{cc}
g_{2 v} & g_{3 v} \\
g_{2 u u} & g_{3 u u}
\end{array}\right|, \quad D_{13}=\left|\begin{array}{cc}
g_{2 v} & g_{3 v} \\
g_{2 u v} & g_{3 u v}
\end{array}\right|, \quad D_{14}=\left|\begin{array}{cc}
g_{2 v} & g_{3 v} \\
g_{2 v v} & g_{3 v v}
\end{array}\right|, \\
D_{23}=\left|\begin{array}{ll}
g_{2 u u} & g_{3 u u} \\
g_{2 u v} & g_{3 u v}
\end{array}\right|, \quad D_{24}=\left|\begin{array}{ll}
g_{2 u u} & g_{3 u u} \\
g_{2 v v} & g_{3 v v}
\end{array}\right|, \quad D_{34}=\left|\begin{array}{ll}
g_{2 u v} & g_{3 u v} \\
g_{2 v v} & g_{3 v v}
\end{array}\right| .
\end{array}
$$

It is obvious that $D_{12}, D_{13}, D_{14}=0$ at 0 . The fact that it is not a Whitney umbrella is also equivalent to $D_{34}=0$ at 0 . Hence, it is non-flat if and only if either $D_{23} \neq 0$ or $D_{24} \neq 0$.

THEOREM 4.9. Let $p=0 \in U$ be an isolated non-flat rounding of rank one of the smooth map $g: U \subset \boldsymbol{R}^{2} \rightarrow \boldsymbol{R}^{3}$ given by $g(u, v)=\left(u, g_{2}(u, v), g_{3}(u, v)\right)$, with $g_{2}, g_{3} \in$ $\langle u, v\rangle^{2}$. Then,

$$
\operatorname{ind}_{\mathcal{R}}(g, p)= \begin{cases}-\operatorname{sign}\left(D_{23}\right) \operatorname{deg}\left\{\left(P_{v}, P_{v v}\right) ;\left(\boldsymbol{R}^{2}, p\right) \rightarrow\left(\boldsymbol{R}^{2}, 0\right)\right\} & \text { if } D_{23} \neq 0, \\ -\operatorname{sign}\left(D_{24}\right) \operatorname{deg}\left\{\left(P_{v}, P_{u v}\right) ;\left(\boldsymbol{R}^{2}, p\right) \rightarrow\left(\boldsymbol{R}^{2}, 0\right)\right\} & \text { if } D_{24} \neq 0 .\end{cases}
$$

Proof. By definition, we have that $\operatorname{ind}_{\mathcal{R}}(g, 0)=\operatorname{deg}\left\{\theta ;\left(\boldsymbol{R}^{5}, 0\right) \rightarrow\left(\boldsymbol{R}^{5}, 0\right)\right\}$, where $\theta$ is given by

$$
\left(\begin{array}{l}
\theta_{1} \\
\theta_{2} \\
\theta_{3} \\
\theta_{4} \\
\theta_{5}
\end{array}\right)=\left(\begin{array}{ccc}
1 & g_{2 u} & g_{3 u} \\
0 & g_{2 v} & g_{3 v} \\
0 & g_{2 u u} & g_{3 u u} \\
0 & g_{2 u v} & g_{3 u v} \\
0 & g_{2 v v} & g_{3 v v}
\end{array}\right)\left(\begin{array}{c}
x \\
y \\
z
\end{array}\right)+\left(\begin{array}{c}
0 \\
0 \\
E \\
F \\
G
\end{array}\right) .
$$

Assume that $D_{23} \neq 0$ at 0 . We can define new coordinates $\tilde{x}, \tilde{y}, \tilde{z}$ by

$$
\left(\begin{array}{c}
\tilde{x} \\
\tilde{y} \\
\tilde{z}
\end{array}\right)=\left(\begin{array}{ccc}
1 & g_{2 u} & g_{3 u} \\
0 & g_{2 u u} & g_{3 u u} \\
0 & g_{2 u v} & g_{3 u v}
\end{array}\right)\left(\begin{array}{l}
x \\
y \\
z
\end{array}\right)+\left(\begin{array}{l}
0 \\
E \\
F
\end{array}\right)
$$

Then, it follows that $\operatorname{deg} \theta=\operatorname{sign}\left(D_{23}\right) \operatorname{deg} \tilde{\theta}$, where $\tilde{\theta}$ is the composite of $\theta$ with such a coordinate change. A simple computation gives that

$$
\tilde{\theta}(\tilde{x}, \tilde{y}, \tilde{z}, u, v)=\left(\tilde{x}, \frac{P_{v v}}{D_{23}}+h_{1}, \tilde{y}, \tilde{z}, \frac{P_{v}}{D_{23}}+h_{2}\right),
$$

where $h_{1}, h_{2} \in\langle\tilde{x}, \tilde{y}, \tilde{z}\rangle$. In particular, we get that

$$
\operatorname{deg} \tilde{\theta}=\operatorname{deg}\left\{\left(\frac{P_{v v}}{D_{23}}, \frac{P_{v}}{D_{23}}\right) ;\left(\boldsymbol{R}^{2}, 0\right) \rightarrow\left(\boldsymbol{R}^{2}, 0\right)\right\}=-\operatorname{deg}\left\{\left(P_{v}, P_{v v}\right) ;\left(\boldsymbol{R}^{2}, 0\right) \rightarrow\left(\boldsymbol{R}^{2}, 0\right)\right\} .
$$

The case $D_{24} \neq 0$ is analogous. 
REMARK 4.10. When $D_{23} \neq 0$, we can use (4.1) and (4.2) in order to obtain $P_{u v}$ in terms of $P_{v}$ and $P_{v v}$. In fact, it follows that $P_{u v}=\left(D_{12} P_{v}+D_{24} P_{v v}\right) / D_{23}$, which implies that

$$
\operatorname{ind}_{\mathcal{P}}(g, p)=-\frac{1}{2} \operatorname{deg}\left\{\left(P_{u v}, P_{v v}\right)\right\}=-\frac{1}{2} \operatorname{sign}\left(D_{23}\right) \operatorname{deg}\left\{\left(D_{12} P_{v}, P_{v v}\right)\right\} .
$$

Analogously, if $D_{24} \neq 0$, we get $P_{u u}=\left(D_{14} P_{v}+D_{34} P_{u v}\right) / D_{24}$, which gives

$$
\operatorname{ind}_{\mathcal{P}}(g, p)=-\frac{1}{2} \operatorname{deg}\left\{\left(P_{u u}, P_{u v}\right)\right\}=-\frac{1}{2} \operatorname{sign}\left(D_{24}\right) \operatorname{deg}\left\{\left(D_{14} P_{v}, P_{u v}\right)\right\} .
$$

Note that $D_{12}=D_{14}=0$ at 0 , so that they have a relevant contribution to the mapping degree of such maps.

4.3.3. Rank zero case. In the rank zero case, it is possible to obtain a similar result. Note that a rank zero rounding of a map $g: U \subset \boldsymbol{R}^{2} \rightarrow \boldsymbol{R}^{3}$ is non-flat if and only if

$$
D=\left|\begin{array}{lll}
g_{1 u u} & g_{1 u v} & g_{1 v v} \\
g_{2 u u} & g_{2 u v} & g_{2 v v} \\
g_{3 u u} & g_{3 u v} & g_{3 u v}
\end{array}\right|
$$

is not zero at $p$. The proof of the following theorem is omitted, since it can be obtained by using the same argument as in the proof of Theorem 4.9.

THEOREM 4.11. Let $g: U \subset \boldsymbol{R}^{2} \rightarrow \boldsymbol{R}^{3}$ be a smooth map and let $p \in U$ be an isolated non-flat rounding of rank zero of $g$. Then

$$
\operatorname{ind}_{\mathcal{R}}(g, p)=-\operatorname{sign}(D) \operatorname{deg}\left\{\left(P_{u}, P_{v}\right) ;\left(\boldsymbol{R}^{2}, p\right) \rightarrow\left(\boldsymbol{R}^{2}, 0\right)\right\} .
$$

EXAMPle 4.12. Consider the map $g: \boldsymbol{R}^{2} \rightarrow \boldsymbol{R}^{3}$ defined by $(u, v) \mapsto\left(u^{2}, u v, v^{2}\right)$. This map is generically two to one. If we take a deformation of this map, there is (at least) one Whitney umbrella near 0 . On the other hand, a simple computation shows that the mapping degree of $\left(P_{u}, P_{v}\right)$ is -1 and hence ind $\operatorname{in}_{\mathcal{R}}(g, 0)=1$. We can conclude that there exists at least one umbilic point near 0 in any deformation of $g$. On the other hand, it is not difficult to see that the mapping degree of $\left(P_{u v}, P_{v v}\right)$ is -2 so that $\operatorname{ind}_{\mathcal{P}}(g, 0)=1$.

In fact, we can consider the generic deformation $g_{\lambda}(u, v)=\left(u^{2}+\lambda v, u v, v^{2}+\lambda u\right)$. For $\lambda \neq 0$ small enough, $g_{\lambda}$ has just one umbilic of type $D_{1}$ with positive principal curvature and one Whitney umbrella near the origin (see Figure 5(a)). However, if we take a different

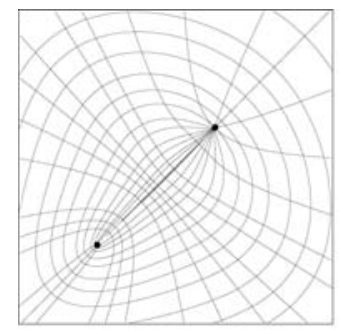

(a)

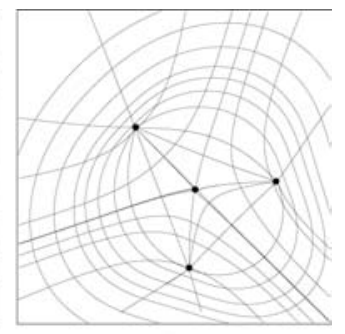

(b)

FIGURE 5. 
generic deformation $g_{\lambda}(u, v)=\left(u^{2}+\lambda v, u v+\lambda u-\lambda v, v^{2}-\lambda u\right)$, this deformation has one umbilic of type $D_{3}$ with negative principal curvature and three Whitney umbrellas (see Figure 5(b)).

5. Complex coordinates. In this section, we discuss the possible values for the index of an isolated umbilic of a regular surface in terms of the coefficients of the Monge normal form:

$$
g=\left(g_{1}, g_{2}, g_{3}\right): \boldsymbol{R}^{2} \rightarrow \boldsymbol{R}^{3}, \quad(u, v) \mapsto(u, v, w(u, v)) .
$$

To simplify notation, we identify $\boldsymbol{R}^{2}$ with $\boldsymbol{C}$ by introducing the complex coordinate $z$ defined by $z=u+i v$.

THEOREM 5.1. We assume that 0 is an umbilic. Then we have

$$
\operatorname{ind}_{\mathcal{P}}(g, 0)=-\frac{1}{2} \operatorname{deg}\left\{\Phi ;(\boldsymbol{C}, 0) \rightarrow(\boldsymbol{C}, 0), \quad z \mapsto w_{z z}\left(\frac{1}{2}+w_{z} w_{\bar{z}}\right)-w_{z \bar{z}} w_{z}^{2}\right\} .
$$

To prove this formula, we recall some basic facts about complex coordinate. We set $z=u+i v, \bar{z}=u-i v$ and

$$
d z=d u+i d v, \quad d \bar{z}=d u-i d v, \quad \frac{\partial}{\partial u}=\frac{\partial}{\partial z}+\frac{\partial}{\partial \bar{z}}, \quad \frac{\partial}{\partial v}=i\left(\frac{\partial}{\partial z}-\frac{\partial}{\partial \bar{z}}\right) .
$$

Then, for any function $f=f(u, v)$, we easily obtain the following relations:

$$
\left(\begin{array}{c}
f_{u} \\
f_{v} \\
f_{u u} \\
f_{u v} \\
f_{v v}
\end{array}\right)=B\left(\begin{array}{c}
f_{z} \\
f_{\bar{z}} \\
f_{z z} \\
f_{z \bar{z}} \\
f_{\bar{z} \bar{z}}
\end{array}\right) \text { where } B=\left(\begin{array}{ccccc}
1 & 1 & 0 & 0 & 0 \\
i & -i & 0 & 0 & 0 \\
0 & 0 & 1 & 2 & 1 \\
0 & 0 & i & 0 & -i \\
0 & 0 & -1 & 2 & -1
\end{array}\right) .
$$

We can write the first fundamental form in terms of $z$ and $\bar{z}$ in the following form:

$$
\mathrm{I}=\left(d g_{1}\right)^{2}+\left(d g_{2}\right)^{2}+\left(d g_{3}\right)^{2}=E_{\boldsymbol{C}} d z^{2}+2 F_{\boldsymbol{C}} d z d \bar{z}+G_{\boldsymbol{C}} d \bar{z}^{2},
$$

where $E_{\boldsymbol{C}}=g_{1}^{2}+g_{2}^{2}+g_{3}^{2}, F_{\boldsymbol{C}}=g_{1 z} g_{1 \bar{z}}+g_{2_{z}} g_{2 \bar{z}}+g_{3 z} g_{3 \bar{z}}, G_{\boldsymbol{C}}=g_{1} \frac{2}{z}+g_{2} \frac{2}{\bar{z}}+g_{3} \frac{2}{\bar{z}}$. Now we consider the matrix

$$
\left(\begin{array}{cccc}
g_{1 z} & g_{2 z} & g_{3 z} & 0 \\
g_{1 \bar{z}} & g_{2 \bar{z}} & g_{3 \bar{z}} & 0 \\
g_{1_{z z}} & g_{2 z z} & g_{3 z z} & E_{\boldsymbol{C}} \\
g_{1 z \bar{z}} & g_{2 z \bar{z}} & g_{3 z \bar{z}} & F_{\boldsymbol{C}} \\
g_{1 \bar{z} \bar{z}} & g_{2 \bar{z} \bar{z}} & g_{3 \bar{z} \bar{z}} & G_{\boldsymbol{C}}
\end{array}\right)=B^{-1}\left(\begin{array}{cccc}
g_{1 u} & g_{2 u} & g_{3 u} & 0 \\
g_{1 v} & g_{2 v} & g_{3 v} & 0 \\
g_{1 u u} & g_{2 u u} & g_{3 u u} & E \\
g_{1 u v} & g_{2 u v} & g_{3 u v} & F \\
g_{1 v v} & g_{2 v v} & g_{3 v v} & G
\end{array}\right)
$$

and observe the following relations:

$$
P_{\bar{z} \bar{z}}:=\left|\begin{array}{cccc}
g_{1 z} & g_{2_{z}} & g_{3 z} & 0 \\
g_{1 \bar{z}} & g_{2 \bar{z}} & g_{3 \bar{z}} & 0 \\
g_{1_{z z}} & g_{2_{z z}} & g_{3_{z z}} & E_{\boldsymbol{C}} \\
g_{1_{z \bar{z}}} & g_{2_{z \bar{z}}} & g_{3_{z \bar{z}}} & F_{\boldsymbol{C}}
\end{array}\right|=\frac{P_{u u}-P_{v v}-i P_{u v}}{16}=\frac{F P_{u v}-(E+G) P_{v v}+i E P_{u v}}{16 E} .
$$

For the last equality we use (4.2). 
Proof (Proof of Theorem 5.1). In the above formula, we have

$$
P_{\bar{z} \bar{z}}=\frac{1}{16 E}\left(P_{u v}, P_{v v}\right)\left(\begin{array}{cc}
F & E \\
-(E+G) & 0
\end{array}\right)
$$

and we conclude that $\operatorname{deg} P_{\bar{z} \bar{z}}=\operatorname{deg}\left(P_{u v}, P_{v v}\right)$, which allows us to compute the index of an isolated umbilic by Theorem 4.5. We thus complete the proof, since

$$
P_{\bar{z} \bar{z}}=\left|\begin{array}{cccc}
1 / 2 & -i / 2 & w_{z} & 0 \\
1 / 2 & i / 2 & w_{\bar{z}} & 0 \\
0 & 0 & w_{z z} & w_{z}^{2} \\
0 & 0 & w_{z \bar{z}} & 1 / 2+w_{z} w_{\bar{z}}
\end{array}\right|=\frac{i}{2}\left(w_{z z}\left(\frac{1}{2}+w_{z} w_{\bar{z}}\right)-w_{z \bar{z}} w_{z}^{2}\right) .
$$

Now we assume that

$$
w=\frac{k}{2} z \bar{z}+\frac{1}{6} C+\frac{1}{24} Q+\frac{1}{120} U+\text { higher order terms },
$$

where $k$ is the principal curvature at 0 and

$$
\begin{gathered}
C=\alpha z^{3}+3 \bar{\beta} z^{2} \bar{z}+3 \beta z \bar{z}^{2}+\bar{\alpha} \bar{z}^{3}, \\
Q=\gamma z^{4}+4 \bar{\delta} z^{3} \bar{z}+6 c z^{2} \bar{z}^{2}+4 \delta z \bar{z}^{3}+\bar{\gamma} \bar{z}^{4},
\end{gathered}
$$

and $U$ is a homogeneous polynomial of degree five. Then we conclude that

$$
\Phi=\frac{1}{12} C_{z z}+\frac{1}{8}\left(\frac{1}{6} Q_{z z}-k^{3} \bar{z}^{2}\right)+\frac{1}{4}\left(\frac{1}{30} U_{z z}+\frac{1}{6} k^{2} z \bar{z} C_{z z}-\frac{1}{3} k^{2} \bar{z} C_{z}-k^{2} \bar{z}^{2} C_{z \bar{z}}\right)+\cdots
$$

and the Jacobian of $\Phi$ is equal to

$$
\left|\Phi_{z}\right|^{2}-\left|\Phi_{\bar{z}}\right|^{2}=\frac{1}{4}\left(|\alpha|^{2}-|\beta|^{2}\right)+\cdots
$$

Now we recover the following well-known fact by Theorem 5.1.

Proposition 5.2. If $|\alpha|>|\beta|$ (resp. $|\alpha|<|\beta|)$, then $\operatorname{ind}_{\mathcal{P}}(g, 0)=-(1 / 2)$ (resp. $1 / 2)$.

These cases correspond to the generic cases if

$$
\alpha z^{3}+\bar{\beta} z^{2} \bar{z}-\beta z \bar{z}^{2}-\bar{\alpha} \bar{z}^{3}
$$

has no multiple zeros (see [25, 12.4], [3, Section 2]).

Proposition 5.3. Consider the Monge normal form (5.1). We assume that

$$
w=\frac{k}{2} z \bar{z}+H+\text { higher order terms, }
$$

where $H$ is a non-zero homogeneous polynomial of degree $n$ with $n \geq 3$. Then:

- if $n \geq 5$ and $k \neq 0$, then $\operatorname{ind}_{\mathcal{P}}(g, 0)=1$;

- if $n \geq 5$ and if the map $H_{z z}:(\boldsymbol{C}, 0) \rightarrow(\boldsymbol{C}, 0)$ is finite, then $\operatorname{ind}_{\mathcal{P}}(g, 0) \leq 1$;

- if $w$ is analytic and $n \neq 4$, then $\left|\operatorname{ind}_{\mathcal{P}}(g, 0)\right| \leq(n-2) / 2$. 
PROOF. We easily obtain that

$$
\Phi(z)=(1 / 2) H_{z z}-(1 / 8) k^{3} \bar{z}^{2}+\cdots,
$$

which shows the first assertion by Theorem 5.1. Since $H_{z z}$ is not identically zero, we may assume that the imaginary part of $H_{z z}$ is not identically zero. We assume that $\Phi$ is a finite map and $w$ is analytic. Then the image of oriented circle $\{z \in C ;|z|=\varepsilon\}$ cut the real axis at most 2(n-2)-times, which implies $|\operatorname{deg} \Phi| \leq n-2$. This shows the last assertion.

We show the second assertion. We assume that $H_{z z}:(\boldsymbol{C}, 0) \rightarrow(\boldsymbol{C}, 0)$ is finite. Since $\operatorname{deg} H_{z z}=\operatorname{deg} \Phi$, it is enough to show that deg $H_{z z} \geq-2$. We define a real-valued function $h(\theta)$ by $H\left(r e^{i \theta}\right)=r^{n} h(\theta)$. Let $a_{+}$(resp. $a_{-}$) denote the number of positive local maximum (resp. minimum) of $h$. We easily see $a_{+} \geq a_{-}$. We define the map $G$ by

$$
G:(\boldsymbol{C}, 0) \rightarrow(\boldsymbol{C}, 0), \quad r e^{i \theta} \mapsto r^{n-2}\left(\left(n(n-2) h(\theta)-h^{\prime \prime}(\theta)\right)+i 2(n-1) h^{\prime}(\theta)\right) .
$$

Since $H_{z z}=(1 / 4) e^{-2 i \theta} G$, we have $\operatorname{deg} H_{z z}=\operatorname{deg} G-2$ and it is enough to show that $\operatorname{deg} G \geq 0$. Consider the set $Z=S^{1} \cap G^{-1}\{x \geq 0, y=0\}$. Each point of $Z$ is a critical point of $h$ with $n(n-2) h-h^{\prime \prime}>0$. Let $b_{+}, b_{-}$denote the numbers of local maximums and minimums of $h$ in the region $n(n-2) h-h^{\prime \prime}>0$. Since $a_{+} \leq b_{+}, a_{-} \geq b_{-}$, we obtain $\operatorname{deg} G=b_{+}-b_{-} \geq a_{+}-a_{-} \geq 0$.

Proposition 5.4. Consider the Monge normal form (5.1) with (5.2). Assume that $C$ is identically zero, and $\left(\gamma, \delta, c_{1}\right) \neq(0,0,0)$ where $c_{1}=c-k^{3} / 2$. Set

$$
R=\left|\begin{array}{cccc}
\gamma & 2 \bar{\delta} & c_{1} & 0 \\
0 & \gamma & 2 \bar{\delta} & c_{1} \\
c_{1} & 2 \delta & \bar{\gamma} & 0 \\
0 & c_{1} & 2 \delta & \bar{\gamma}
\end{array}\right|=c_{1}^{4}-2\left(|\gamma|^{2}+2|\delta|^{2}\right) c_{1}^{2}+8 \Re\left(\gamma \delta^{2}\right) c_{1}+|\gamma|^{2}\left(|\gamma|^{2}-4|\delta|^{2}\right) .
$$

We choose $\theta_{1}$ so that $\gamma_{1}=\gamma e^{4 \theta_{1} i}$ is a non-negative real number. Define real numbers $p, q$ by $\delta_{1}=\delta e^{-2 \theta_{1} i}=p+q i$. If 0 is an isolated umbilic, then we have the following:

(1) $\left|\operatorname{ind}_{\mathcal{P}}(g, 0)\right| \leq 1$;

(2) if $R>0,\left|c_{1}\right|<\gamma_{1}$ (resp. $\left.\left|c_{1}\right|>\gamma_{1}\right)$, then $\operatorname{ind}_{\mathcal{P}}(g, 0)=-1$ (resp. 1);

(3) if $R<0$, then $\operatorname{ind}_{\mathcal{P}}(g, 0)=0$;

(4) if $R=0$ and $c_{1}^{2}-\gamma_{1}^{2} \neq 0$, then $\left|\operatorname{ind}_{\mathcal{P}}(g, 0)\right| \leq 1 / 2$;

(5) if $R=c_{1}^{2}-\gamma_{1}^{2}=0$, then $\operatorname{ind}_{\mathcal{P}}(g, 0)=0$ except for the following two cases:

$$
\begin{aligned}
& \text { (a) } c_{1}-\gamma_{1}=0, q=0,|p|<\left|c_{1}\right| \text {; } \\
& \text { (b) } c_{1}+\gamma_{1}=0, p=0,|q|>\left|c_{1}\right| \text {. }
\end{aligned}
$$

Proof. (1) We easily obtain that

$$
\Phi(z)=\left(Q_{z z} / 6-k^{3} \bar{z}^{2}\right) / 8+\cdots=\left(\gamma z^{2}+2 \bar{\delta} z \bar{z}+c_{1} \bar{z}^{2}\right) / 4+\cdots,
$$

and $\Phi$ has rank zero at 0 . If $\left(\gamma, \delta, c_{1}\right) \neq(0,0,0)$, then the initial form is of degree 2 and $|\operatorname{deg} \Phi| \leq 2$. This implies (1).

(2) and (3) Observe that $R$ is the resultant of the initial form of $\Phi$ and $\bar{\Phi}$. If $R \neq 0$, then the initial part is a homogeneous map of degree two with finite multiplicity. We may assume that $\gamma \neq 0$. This implies that $\Phi$ is semi-quasi-homogeneous and the mapping degree 


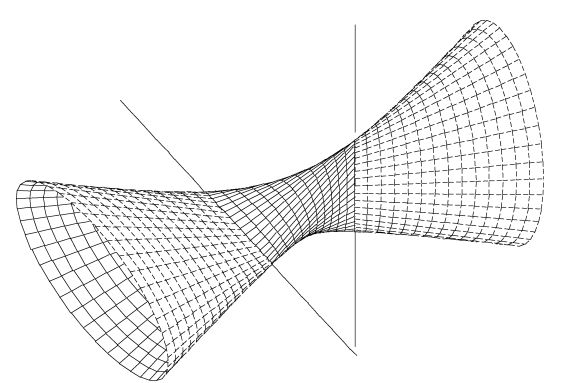

FIGURE 6.

of $\Phi$ is equal to the mapping degree of its initial part (see [4]). Moreover, the initial part has multiplicity 4 and according to [6], the mapping degree of $\Phi$ will be \pm 2 or 0 .

Setting $z=z_{1} e^{\theta_{1} i}$, we have

$$
\begin{aligned}
& Q=\gamma_{1} z_{1}^{4}+4 \overline{\delta_{1}} z_{1}^{3} \overline{z_{1}}+6 c z_{1}^{2}{\overline{z_{1}}}^{2}+4 \delta_{1} z_{1} \bar{z}_{1}^{3}+{\overline{\gamma_{1}}}_{z_{1}}^{4}, \\
& R=\left(c_{1}^{2}-\gamma_{1}^{2}\right)^{2} / 4-\left(c_{1}-\gamma_{1}\right)^{2} p^{2}-\left(c_{1}+\gamma_{1}\right)^{2} q^{2} .
\end{aligned}
$$

By looking at $c_{1}, \gamma_{1}, p, q$ as coordinates in $\boldsymbol{R}^{4}$, it is not difficult to see that the equation $R=0$ separates the region $\left\{\gamma_{1}>0\right\}$ of $\boldsymbol{R}^{4}$ in four connected components, corresponding to the following regions: (1) $R>0,\left|c_{1}\right|<\gamma_{1}$; (2) $R>0, c_{1}>\gamma_{1}$; (3) $R>0, c_{1}<-\gamma_{1}$; and (4) $R<0$. Since the mapping degree will be constant on each of these regions, it is enough to compute the value of the mapping degree for a particular point $\left(c_{1}, \gamma_{1}, p, q\right)$ in each region. In Figure 6 , we show the zero locus of $R$ with $\gamma_{1}=1$ in the $\left(c_{1}, p, q\right)$-space.

In the region (1), we take $c_{1}=p=q=0$ and $\gamma_{1}>0$. It follows that $\Phi=\gamma_{1} z^{2}+\cdots$ so that the mapping degree of $\Phi$ is 2 .

In the regions (2) and (3), we take $\gamma_{1}=p=q=0$ and $c_{1} \neq 0$, so that $\Phi=c_{1} \bar{z}^{2} / 4+\cdots$ and the mapping degree is -2 .

Finally, in the region $R<0$, we take $c_{1}=\gamma_{1}>0, p=0$ and $q \neq 0$. Then $\Phi=$ $(1 / 2)\left(\gamma_{1}\left(u^{2}-v^{2}\right), q\left(u^{2}+v^{2}\right)\right)+\cdots$, which has mapping degree 0 .

(4) We first assume that $c_{1}^{2}-\gamma_{1}^{2} \neq 0$. Then the condition $R=0$ is equivalent to

$$
\frac{p^{2}}{\left(\left(c_{1}+\gamma_{1}\right) / 2\right)^{2}}+\frac{q^{2}}{\left(\left(c_{1}-\gamma_{1}\right) / 2\right)^{2}}=1,
$$

which defines a surface parameterized by

$$
p=\frac{c_{1}+\gamma_{1}}{2} \cos \theta_{2}, \quad q=\frac{c_{1}-\gamma_{1}}{2} \sin \theta_{2}, \quad 0 \leq \theta_{2} \leq 2 \pi .
$$

Then, setting $z_{1}=u_{1}+v_{1} i$, we obtain that

$$
\Phi=\frac{1}{4}\left(\gamma_{1} z_{1}^{2}+2 \delta_{1} z_{1} \overline{z_{1}}+c_{1}{\overline{z_{1}}}^{2}\right)+\cdots=\frac{\left(c_{1}+\gamma_{1}\right) g_{1}-\left(c_{1}-\gamma_{1}\right) g_{2} i}{2} g_{0}+\cdots,
$$

where $g_{0}=u_{1} \cos \left(\theta_{2} / 2\right)+v_{1} \sin \left(\theta_{2} / 2\right), g_{1}=u_{1} \cos \left(\theta_{2} / 2\right)-v_{1} \sin \left(\theta_{2} / 2\right), g_{2}=u_{1} \sin \left(\theta_{2} / 2\right)+$ $v_{1} \cos \left(\theta_{2} / 2\right)$. If we know the image of the line $\left\{g_{0}=0\right\}$ by $\Phi$, we can decide the mapping 
degree. Case-by-case analysis shows that if $\Phi$ is finite, then $|\operatorname{deg} \Phi| \leq 1$, and we complete the proof of (4).

(5) Assume next that $R=0$ and $c_{1}^{2}-\gamma_{1}^{2}=0$. Then $c_{1}-\gamma_{1}=0$ or $c_{1}+\gamma_{1}=0$. If $c_{1}-\gamma_{1}=0$ (resp. $c_{1}+\gamma_{1}=0$ ), then $q=0$ (resp. $p=0$ ) since $R=0$. We then obtain

$$
\Phi=\frac{c_{1}+p}{2} u_{1}^{2}-\frac{c_{1}-p}{2} v_{1}^{2}+\cdots \quad\left(\operatorname{resp} . \Phi=-\left(\frac{q}{2} u_{1}^{2}+c_{1} u_{1} v_{1}+\frac{q}{2} v_{1}^{2}\right) i+\cdots\right) .
$$

The proof follows from the fact that the quadratic part of $\Phi$ is definite and, hence, the mapping degree of $\Phi$ becomes zero.

REMARK 5.5. Assume that $C$ is identically zero and $\gamma=\delta=c_{1}=0$. Then $w$ is given by

$$
w=\frac{k}{2} z \bar{z}-\frac{k^{3}}{8} z^{2} \bar{z}^{2}+H+\cdots
$$

where $H$ is a non-zero homogeneous polynomial of degree $n \geq 5$. If $\Phi=(1 / 2) H_{z z}+\cdots$ is a finite map, then 0 is an isolated umbilic and $(2-n) / 2 \leq \operatorname{ind}_{\mathcal{P}}(g, 0) \leq 1$, by a discussion similar to the proof of Proposition 5.3.

EXAMPLE 5.6. We finish this section by showing that it is possible to construct surfaces with umbilics of any index $\leq 1$. Let $n$ be an integer with $n \geq 2$. Consider the surface defined by $w=\left(z^{n}+\bar{z}^{n}\right) / 2$. Then we have

$$
\Phi(z)=\frac{n(n-1)}{2} z^{n-2}+\cdots
$$

and $\operatorname{ind}_{\mathcal{P}}(g, 0)=-(n-2) / 2$.

Similarly we consider the surface defined by $w=(z \bar{z})^{m}\left(z^{n}+\bar{z}^{n}\right) / 2$. Then

$$
\Phi(z)=(m+n)(m+n-1)(z \bar{z})^{m-2} \bar{z}^{2}\left(z^{n}+\frac{m(m-1)}{(m+n)(m+n-1)} \bar{z}^{n}\right)+\cdots
$$

which is a map of mapping degree $n-2$. Thus, we get $\operatorname{ind}_{\mathcal{P}}(g, 0)=-(n-2) / 2$.

6. Surfaces in $\boldsymbol{R}^{4}$. In this last section, we consider a smooth map $g: U \subset \boldsymbol{R}^{2} \rightarrow \boldsymbol{R}^{4}$ given by $g(u, v)=\left(g_{1}(u, v), g_{2}(u, v), g_{3}(u, v), g_{4}(u, v)\right)$, which defines a smooth surface in $\boldsymbol{R}^{4}$ (possibly with singularities). We look at the 2 -flattenings of $g$, which correspond to points where the matrix

has rank $<4$.

$$
F(g, t)=\left(\begin{array}{cccc}
g_{1 u} & g_{2 u} & g_{3 u} & g_{4 u} \\
g_{1 v} & g_{2 v} & g_{3 v} & g_{4 v} \\
g_{1} u u & g_{2 u u} & g_{3 u u} & g_{4 u u} \\
g_{1 u v} & g_{2 u v} & g_{3 u v} & g_{4 u v} \\
g_{1 v v} & g_{2 v v} & g_{3 v v} & g_{4 v v}
\end{array}\right)
$$

On the other hand, the geometry of regular surfaces in $\boldsymbol{R}^{4}$ has been studied by several authors. The local second-order invariants of these surfaces were described by Little [16] in the following way: we have a first fundamental form

$$
\mathrm{I}=E d u^{2}+2 F d u d v+G d v^{2}, \quad E=\left\langle g_{u}, g_{u}\right\rangle, \quad F=\left\langle g_{u}, g_{v}\right\rangle, \quad G=\left\langle g_{v}, g_{v}\right\rangle,
$$


and two second fundamental forms

$$
\begin{gathered}
\mathrm{II}_{i}=L_{i} d u^{2}+2 M_{i} d u d v+N_{i} d v^{2}, \quad L_{i}=\left\langle g_{u u}, e_{i}\right\rangle, \\
M_{i}=\left\langle g_{u v}, e_{i}\right\rangle, \quad N_{i}=\left\langle g_{v v}, e_{i}\right\rangle, \quad i=1,2,
\end{gathered}
$$

with $\left\{e_{1}, e_{2}\right\}$ an orthonormal basis of the normal plane to the surface at the point (this only makes sense at the regular points of $g$ ). Associated with these quadratic forms we have the following functions:

$$
\Delta=\frac{1}{4}\left|\begin{array}{cccc}
L_{1} & 2 M_{1} & N_{1} & 0 \\
L_{2} & 2 M_{2} & N_{2} & 0 \\
0 & L_{1} & 2 M_{1} & N_{1} \\
0 & L_{2} & 2 M_{2} & N_{2}
\end{array}\right|, \quad K=L_{1} N_{1}-M_{1}^{2}+L_{2} N_{2}-M_{2}^{2},
$$

and the matrix

$$
\alpha=\left(\begin{array}{lll}
L_{1} & M_{1} & N_{1} \\
L_{2} & M_{2} & N_{2}
\end{array}\right) .
$$

That is, $\Delta$ is the resultant of the two second fundamental forms; $K$ is called the Gaussian curvature of $g$ and it is shown in [16] that $\Delta, K$ and the rank of $\alpha$ are coordinate independent. Then, we can classify the points of the surface in terms of these functions as follows.

(1) If $\Delta<0$, the point is said to be hyperbolic.

(2) If $\Delta>0$, the point is said to be elliptic.

(3) If $\Delta=0$, the point is said to be parabolic.

(4) If $\alpha$ has rank $<2$, the point is said to be an inflection. Moreover, we have the following types of inflection:

(a) if $K>0$, it is an inflection of imaginary type;

(b) if $K<0$, it is an inflection of real type;

(c) if $K=0$, it is an inflection of flat type.

PROPOSITION 6.1. A regular point of a smooth surface in $\boldsymbol{R}^{4}$ is a flattening if and only if it is an inflection.

ProOF. Since $g$ is regular, we may assume that our point is the origin of $\boldsymbol{R}^{2}$ and that $g$ is given by $g(u, v)=\left(u, v, g_{3}(u, v), g_{4}(u, v)\right)$, with

$$
g_{3}=\left(a_{1} u^{2}+2 b_{1} u v+c_{1} v^{2}\right) / 2+\cdots, \quad g_{4}=\left(a_{2} u^{2}+2 b_{2} u v+c_{2} v^{2}\right) / 2+\cdots .
$$

Then, we have

$$
F(g, 0)=\left(\begin{array}{cccc}
1 & 0 & 0 & 0 \\
0 & 1 & 0 & 0 \\
0 & 0 & a_{1} & a_{2} \\
0 & 0 & b_{1} & b_{2} \\
0 & 0 & c_{1} & c_{2}
\end{array}\right) \quad \text { and } \quad \alpha(0)=\left(\begin{array}{lll}
a_{1} & b_{1} & c_{1} \\
a_{2} & b_{2} & c_{2}
\end{array}\right) .
$$

Here we take $e_{1}=(0,0,1,0)$ and $e_{2}=(0,0,0,1)$ as an orthonormal basis of the normal plane to $g$ at 0 . The result is clear now. 
REMARK 6.2. It follows from the above proof that if $t$ is a regular point of $g$ and the matrix $F(g, t)$ has rank $<3$, then $t$ is an inflection of flat type.

The multiplicity of a flattening $\mu_{\mathcal{F}}(g, p)$ is related to the number of generic inflections that appear in a generic deformation of $g$ near $p$. Recall that it is defined by

$$
\mu_{\mathcal{F}}(g, p)=\operatorname{dim}_{\boldsymbol{R}} C^{\infty}\left(\boldsymbol{R}^{2}, p\right) / \mathcal{F}(g, p),
$$

where $\mathcal{F}(g, p)$ is the ideal generated by $P_{u}, P_{v}, P_{u u}, P_{u v}, P_{v v}$, the maximal minors of $F(g, t)$ :

$$
P_{u}=\operatorname{det}\left(g_{v}, g_{u u}, g_{u v}, g_{v v}\right), \quad P_{v}=\operatorname{det}\left(g_{u}, g_{u u}, g_{u v}, g_{v v}\right),
$$

$P_{u u}=\operatorname{det}\left(g_{u}, g_{v}, g_{u v}, g_{v v}\right), \quad P_{u v}=\operatorname{det}\left(g_{u}, g_{v}, g_{u u}, g_{v v}\right), \quad P_{v v}=\operatorname{det}\left(g_{u}, g_{v}, g_{u u}, g_{u v}\right)$.

Moreover, the relations between these five generators are given by:

$$
g_{i u} P_{u}-g_{i v} P_{v}+g_{i u u} P_{u u}-g_{i u v} P_{u v}+g_{i v v} P_{v v}=0 \quad(i=1,2,3,4) .
$$

When rank $F(g, p)=3$, we can consider the index $\operatorname{ind}_{\mathcal{F}}(g, p)$, which is defined as the mapping degree of $\theta:\left(S^{3} \times \boldsymbol{R}^{2},\left(v_{0}, p\right)\right) \rightarrow\left(\boldsymbol{R}^{5}, 0\right)$ given by $\theta(v, t)=F(g, t) v$ and $v_{0} \in S^{3}$ is chosen so that $\left(v_{0}, p\right) \in \Sigma^{2,2}(H)$. Since we have two possible choices for the vector, namely $\pm v_{0}$, the index is well defined up to sign. We have the following possibilities.

(1) Rank 2 case. Since $g$ has rank 2, we see that $g_{u}, g_{v}$ and one of $\left\{g_{u u}, g_{u v}, g_{v v}\right\}$ are linearly independent at $p$. Suppose, for instance, that $g_{u}, g_{v}, g_{u u}$ are linearly independent. Then, $P_{u v}, P_{v v}$ generate the ideal $\mathcal{F}(g, p)$ and

$$
\left|\operatorname{ind}_{\mathcal{F}}(g, p)\right|=\left|\operatorname{deg}\left(P_{u v}, P_{v v}\right)\right| .
$$

(2) Rank 1 case. In this case one of $\left\{g_{u}, g_{v}\right\}$ and two of $\left\{g_{u u}, g_{u v}, g_{v v}\right\}$ are linearly independent at $p$. Suppose, for instance, that $g_{u}, g_{u u}, g_{u v}$ are linearly independent. Then, $P_{v}, P_{v v}$ generate the ideal $\mathcal{F}(g, p)$ and

$$
\left|\operatorname{ind}_{\mathcal{F}}(g, p)\right|=\left|\operatorname{deg}\left(P_{v}, P_{u v}\right)\right| \text {. }
$$

(3) Rank 0 case. Now the only possibility is that $g_{u u}, g_{u v}, g_{v v}$ are linearly independent at $p$. Then, $P_{u}, P_{v}$ generate the ideal $\mathcal{F}(g, p)$ and

$$
\left|\operatorname{ind}_{\mathcal{F}}(g, p)\right|=\left|\operatorname{deg}\left(P_{u}, P_{v}\right)\right| \text {. }
$$

As in the case of a surface in $\boldsymbol{R}^{3}$, the index $\operatorname{ind}_{\mathcal{F}}(g, p)$ is related to an index defined by a geometrical line field in the surface, namely the field of asymptotic directions. According to [8], the asymptotic directions at a regular point are given by the quadratic differential equation

$$
\left|\begin{array}{ccccc}
g_{1 u} & g_{2 u} & g_{3 u} & g_{4 u} & 0 \\
g_{1 v} & g_{2 v} & g_{3 v} & g_{4 v} & 0 \\
g_{1 u u} & g_{2 u u} & g_{3 u u} & g_{4 u} & d v^{2} \\
g_{1 u v} & g_{2 u v} & g_{3 u v} & g_{4 u v} & -d u d v \\
g_{1 v v} & g_{2 v v} & g_{3 v v} & g_{4 v v} & d u^{2}
\end{array}\right|=P_{u u} d v^{2}+P_{u v} d u d v+P_{v v} d u^{2}=0
$$


Moreover, the sign of the discriminant of this equation coincides with the sign of $-\Delta$. Thus, at a hyperbolic point there exist exactly two asymptotic directions; at an elliptic point there exist no asymptotic directions; at a parabolic point which is not an inflection, there exists exactly one asymptotic direction; and at an inflection, all of the directions are asymptotic.

Suppose now that $p$ is an isolated inflection of imaginary type. This implies that $\Delta=0$ and $K>0$ at $p$ and that $\Delta<0$ for all $t \neq p$ in a neighborhood of $p$. In particular, we have a pair of asymptotic directions defined in such a neighborhood with an isolated singularity at $p$. The index associated with these asymptotic directions will be denoted by $\operatorname{ind}_{\mathcal{A}}(g, p)$. The configuration of the asymptotic lines at a generic inflection of imaginary type has been obtained recently in [8], where they show that the only possibilities are again the Darbouxian configurations, with index $\pm(1 / 2)$.

Note that if $p$ is an inflection of imaginary type, then the condition $K>0$ ensures that both $\left\{g_{u}, g_{v}, g_{u u}\right\}$ and $\left\{g_{u}, g_{v}, g_{v v}\right\}$ are linearly independent. Moreover, they determine the same oriented 3-plane. That is, we have a canonical choice for the vector $v_{0} \in S^{3}$ such that $\left(v_{0}, p\right) \in \Sigma^{3,3}(H)$. This choice is given by

$$
v_{0}=\frac{g_{u} \wedge g_{v} \wedge g_{u u}}{\left\|g_{u} \wedge g_{v} \wedge g_{u u}\right\|}(p)=\frac{g_{u} \wedge g_{v} \wedge g_{v v}}{\left\|g_{u} \wedge g_{v} \wedge g_{v v}\right\|}(p) .
$$

Thus, in the case of an inflection of imaginary type, we have a well defined index $\operatorname{ind}_{\mathcal{F}}(g, p)$.

THEOREM 6.3. Let $g: U \subset \boldsymbol{R}^{2} \rightarrow \boldsymbol{R}^{4}$ be a smooth map and let $p \in U$ be an isolated inflection of imaginary type of $g$. Then

$$
\operatorname{ind}_{\mathcal{A}}(g, p)=-\frac{1}{2} \operatorname{deg}\left\{\left(P_{u u}, P_{u v}\right)\right\}=-\frac{1}{2} \operatorname{deg}\left\{\left(P_{u v}, P_{v v}\right)\right\}=\frac{1}{2} \operatorname{ind}_{\mathcal{F}}(g, p) .
$$

ProOF. Since in this case both $\left\{g_{u}, g_{v}, g_{u u}\right\}$ and $\left\{g_{u}, g_{v}, g_{v v}\right\}$ are linearly independent, both $\left\{P_{u v}, P_{v v}\right\}$ and $\left\{P_{u u}, P_{u v}\right\}$ generate the ideal $\mathcal{F}(g, p)$. Then, the two first equalities follow from Lemma 4.3. To see the last equality, we will show that it is true for a generic inflection. In the general case, we can show the result by taking a generic deformation of $g$.

If $p$ is a generic inflection, then the multiplicity $\mu_{\mathcal{F}}(g, p)$ is one. Therefore, the map germs $\left(P_{u u}, P_{u v}\right):\left(\boldsymbol{R}^{2}, p\right) \rightarrow\left(\boldsymbol{R}^{2}, 0\right)$ and $\left(P_{u v}, P_{v v}\right):\left(\boldsymbol{R}^{2}, p\right) \rightarrow\left(\boldsymbol{R}^{2}, 0\right)$ are regular.

In general, if $p$ is an inflection, we may assume that $p=0$ and that $g$ is given by the Monge normal form $g(u, v)=\left(u, v, g_{3}(u, v), g_{4}(u, v)\right)$, with

$g_{3}(u, v)=\left(a u^{2}+2 b u v+c v^{2}\right) / 2+\cdots, \quad g_{4}(u, v)=\left(p u^{3}+3 q u^{2} v+3 r u v^{2}+s v^{3}\right) / 6+\cdots$.

Then, $P_{u}=P_{v}=0$ and

$$
\begin{aligned}
& P_{u u}=(b r-c q) u+(b s-c r) v+\cdots, \\
& P_{u v}=(a r-c p) u+(a s-c q) v+\cdots, \\
& P_{v v}=(a q-b p) u+(a r-b q) v+\cdots .
\end{aligned}
$$

Note that if the inflection is of imaginary type, it follows that $K=a c-b^{2}>0$. In particular, $a c>0$. 
We now compute the Jacobian determinants of $\left(P_{u u}, P_{u v}\right)$ and $\left(P_{u v}, P_{v v}\right)$ which give, respectively, $c \Delta$ and $a \Delta$, with

$$
\Delta=a\left(r^{2}-q s\right)+b(p s-r q)+c\left(q^{2}-r p\right) .
$$

Thus, the fact that the inflection is generic implies that $\Delta \neq 0$.

To see the value of $\operatorname{ind}_{\mathcal{F}}(g, 0)$ we have to compute the Jacobian determinant of $\theta:\left(S^{3} \times \boldsymbol{R}^{2},\left(v_{0}, 0\right)\right) \rightarrow \boldsymbol{R}^{5}$ given by $\theta(v, t)=F(g, t) v$, where

$$
v_{0}=\frac{g_{u} \wedge g_{v} \wedge g_{u u}}{\left\|g_{u} \wedge g_{v} \wedge g_{u u}\right\|}(0)
$$

It is not difficult to see that if $a>0$, then $v_{0}=(0,0,0,1)$ and the Jacobian determinant of $\theta$ is $-\Delta$; if $a<0$, then $v_{0}=(0,0,0,-1)$ and the Jacobian determinant of $\theta$ is $\Delta$.

REMARK 6.4. Note that an umbilic of a surface $g$ in $\boldsymbol{R}^{3}$ is carried by the inverse of the stereographic projection $\xi: \boldsymbol{R}^{3} \rightarrow S^{3} \hookrightarrow \boldsymbol{R}^{4}$ into an inflection of the corresponding surface $\xi \circ g$ in $\boldsymbol{R}^{4}$ (see the end of Section 2). Moreover, it follows from Theorem 3 of [18] that the curvature lines of $g$ are carried into the asymptotic lines of $\xi \circ g$. Therefore, if $p$ is an isolated umbilic of $g$, then $p$ is an inflection of imaginary type of $\xi \circ g$ and

$$
\operatorname{ind}_{\mathcal{P}}(g, p)=\operatorname{ind}_{\mathcal{A}}(\xi \circ g, p) \text {. }
$$

Acknowledgments. The authors would like to thank M. C. Romero-Fuster, A. Montesinos-Amilibia and S. Izumiya for helpful comments. The authors also wish to thank the referee for many comments on the earlier version of this paper, and informing the authors about the controversy surrounding the proof of the Carathéodory conjecture.

The first author has been partially supported by Grand-in-Aid in Science 11440017, 15340017. The second author has been partially supported by DGICYT Grant BFM200302037.

\section{REFERENCES}

[ 1 ] V. I. ARNOLD, On the number of flattening points on space curves, in Sină̌s Moscow seminar on dynamical systems, 11-22, Amer. Math. Soc. Transl. Ser. 2 171, Amer. Math. Soc., Providence, R.I., 1996.

[2] L. BATES, A weak counterexample to the Carathéodory conjecture, Differential Geom. Appl. 15 (2001), 7980 .

[ 3 ] J. W. BRUCE AND D. L. Fidal, On binary differential equations and umbilics, Proc. Roy Soc. Edinburgh Sect. A 111 (1989), 147-168.

[4] A. Cima, A. Gasull and J. Torregros A, On the relation between index and multiplicity, J. London Math. Soc. (2) 57 (1998), 757-768.

[ 5 ] G. Darboux, Leçons sur la théorie générales des surfaces, vol. 4, Gauthier-Villars, Paris, 1896.

[6] D. Eisenbud And H. I. Levine, An algebraic formula for the degree of a $C^{\infty}$ map germ, Ann. Math. (2) 106 (1977), 19-44.

[7] R. A. Garcia, C. Gutierrez and J. Sotomayor, Lines of principal curvature around umbilics and Whitney umbrellas, Tohoku Math. J. (2) 52 (2000), 163-172.

[ 8 ] R. A. Garcia, D. K. H. Mochida, M. C. Romero-Fuster and M. A. S. Ruas, Inflection points and topology of surfaces in 4-space, Trans. Amer. Math. Soc. 352 (2000), 3029-3043. 
[9] V. Guíñez, Positive quadratic differential forms and foliations with singularities on surfaces, Trans. Amer. Math. Soc. 309 (1988), 477-502.

[10] C. Gutierrez And J. Sotomayor, Structural stable configurations of lines of principal curvature, Bifurcation, ergodic theory and applications (Dijon, 1981), 195-215, Asterisque 98-99, Soc. Math. France, Paris, 1982.

[11] C. Gutierrez And J. Sotomayor, Lines of principal curvature for mappings with Whitney umbrella singularities, Tohoku Math. J. (2) 38 (1986), 551-559.

[12] M. Hochster And J. A. EAgOn, Cohen-Macaulay rings, invariant theory, and the generic perfection of determinantal loci, Amer. J. Math. 93 (1971), 1020-1058.

[13] H. Hopf, Differential geometry in the large, Lecture Notes in Math. 1000, Springer, New York, 1983.

[14] V. V. IVAnov, An analytic conjecture of Carathéodory, Siberian Math. J. 43 (2002), 251-322.

[15] E. P. LAnE, Projective differential geometry of curves and surfaces, University of Chicago Press, Chicago, Ill., 1932.

[16] J. A. Little, On singularities of submanifolds of higher dimensional Euclidean spaces, Ann. Mat. Pura Appl. (4) 83 (1969), 261-335.

[17] H. Matsumura, Commutative ring theory, Cambridge Stud. Adv. Math. 8, Cambridge Univ. Press, Cambridge, 1986.

[18] D. K. H. Mochida, M. C. Romero-Fuster And M. A. S. Ruas, Osculating hyperplanes and asymptotic directions of codimension two submanifolds of Euclidean spaces, Geom. Dedicata 77 (1999), 305-315.

[19] J. A. Montaldi, Contacts with applications to submanifolds of $\boldsymbol{R}^{n}$, Thesis, University of Liverpool, 1983.

[20] J. A. Montaldi, On contact between submanifolds, Michigan Math. J. 33 (1986), 195-199.

[21] A. Montesinos-Amilibia, Superficies, Computer program available at ftp://topologia.geomet.uv.es/pub/ montesin.

[22] D. Mumford, Algebraic geometry I, Complex projective varieties, Grundlehren Math. Wiss. 221, Springer, New York, 1976

[23] J. J. NuÑo BALlesteros AND M. J. SAIA, Multiplicity of Boardman strata and deformations of map germs, Glasgow Math. J. 40 (1998), 21-32.

[24] I. R. PoRteous, The normal singularities of a submanifold, J. Differential Geom. 5 (1971), 543-564.

[25] I. R. Porteous, Geometric differentiation for the intelligence of curves and surfaces, Cambridge University Press, Cambridge, 1994.

[26] M. C. Romero FUSTER, Stereographical projections and geometric singularities, Mat. Contemp. 12 (1997), $167-182$.

[27] M. C. Romero Fuster, Geometric singularities of curves and surfaces and their stereographical images, Quart. J. Math. Oxford Ser. (2) 49 (1998), 363-373.

[28] M. C. Romero Fuster and E. Sanabria Codesal, Generalized evoultes, vertices and conformal invariants of curves in $\boldsymbol{R}^{n+1}$, Indag. Math. (N. S.) 10 (1999), 297-305.

[29] V. D. SEDYKH, A relationship between Lagrange and Legendre singularities in stereographic projection Mat. Sb. 185 (1994), 123-130 (English transl. Russian Acad. Sci. Sb. Math. 83 (1995), 533-540).

[30] B. Smyth And F. XAVIER, A sharp geometric estimate for the index of an umbilic on a smooth surface, Bull. London Math. Soc. 24 (1992), 176-180.

[31] C. J. TiTUs, A proof of a conjecture of Loewner and of the conjecture of Caratheodory on umbilic points, Acta Math. 131 (1973), 43-77.

[32] C. T. C. WALL, Geometric properties of generic differentiable manifolds, Geometry and topology (Proc. III Latin Amer. School of Math., Inst. Mat. Pura Aplicada CNPq, Rio de Janeiro, 1976), 707-774, Lecture Notes in Math. 597, Springer, New York, 1977. 
DEPARTMENT OF MATHEMATICS

FACULTY OF SCIENCE

SAITAMA UNIVERSITY

255 SHIMO-OKUBO, SAKURA-KU

SAITAMA 338-8570

JAPAN

E-mail address: tfukui@ rimath.saitama-u.ac.jp
DEPARTAMENT DE GEOMETRIA I TOPOLOGIA UNIVERSITAT DE VALÈNCIA

CAMPUS DE BURJASSOT

46100 BURJASSOT

SPAIN

E-mail address: nuno@uv.es 